\title{
Long Range Scattering and Modified Wave Operators for some Hartree Type Equations II ${ }^{*}$
}

\author{
J. Ginibre \\ Laboratoire de Physique Théoriquel] \\ Université de Paris XI, Bâtiment 210, F-91405 Orsay Cedex, France \\ G. Velof \\ Laboratoire d'Analyse Numérique et E.D.P. 3 \\ Université de Paris XI, Bâtiment 425, F-91405 Orsay Cedex, France
}

\begin{abstract}
We study the theory of scattering for a class of Hartree type equations with long range interactions in space dimension $n \geq 3$, including Hartree equations with potential $V(x)=\lambda|x|^{-\gamma}$. For $0<\gamma \leq 1$ we prove the existence of modified wave operators with no size restriction on the data and we determine the asymptotic behaviour in time of solutions in the range of the wave operators, thereby extending the results of a previous paper which covered the range $1 / 2<\gamma<1$.
\end{abstract}

AMS Classification : Primary 35P25. Secondary 35B40, 35Q40, 81 U99.

Key words : Long range scattering, modified wave operators, Hartree equation.

LPT Orsay 99-14

February 1999

*Work supported in part by NATO Collaborative Research Grant 972231.

†Unité Mixte de Recherche (CNRS) UMR 8627.

${ }_{\ddagger}^{\ddagger}$ Permanent address : Dipartimento di Fisica, Università di Bologna and INFN, Sezione di Bologna, Italy.

$\S$ Unité Mixte de Recherche (CNRS) UMR 8628. 


\section{Introduction}

This is the second paper where we study the theory of scattering and more precisely the existence of modified wave operators for a class of long range Hartree type equations

$$
i \partial_{t} u+\frac{1}{2} \Delta u=\widetilde{g}\left(|u|^{2}\right) u
$$

where $u$ is a complex function defined in space time $I^{n+1}, \Delta$ is the Laplacian in $I R^{n}$, and

$$
\widetilde{g}\left(|u|^{2}\right)=\lambda t^{\mu-\gamma} \omega^{\mu-n}|u|^{2}
$$

with $\omega=(-\Delta)^{1 / 2}, \lambda \in I R, 0<\gamma \leq 1$ and $0<\mu<n$. The operator $\omega^{\mu-n}$ can also be represented by the convolution in $x$

$$
\omega^{\mu-n} f=C_{n, \mu}|x|^{-\mu} * f
$$

so that (1.2) is a Hartree type interaction with potential $V(x)=C|x|^{-\mu}$. The more standard Hartree equation corresponds to the case $\gamma=\mu$. In that case, the nonlinearity $\widetilde{g}\left(|u|^{2}\right)$ becomes

$$
\widetilde{g}\left(|u|^{2}\right)=V *|u|^{2}=\lambda|x|^{-\gamma} *|u|^{2}
$$

with a suitable redefinition of $\lambda$.

A large amount of work has been devoted to the theory of scattering for the Hartree equation (1.1) with nonlinearity (1.4) as well as with similar nonlinearities with more general potentials. As in the case of the linear Schrödinger equation, one must distinguish the short range case, corresponding to $\gamma>1$, from the long range case corresponding to $\gamma \leq 1$. In the short range case, it is known that the (ordinary) wave operators exist in suitable function spaces for $\gamma>1$ [11. Furthermore for repulsive interactions, namely for $\lambda \geq 0$, it is known that all solutions in suitable spaces admit asymptotic states in $L^{2}$ for $\gamma>1$, and that asymptotic completeness holds for $\gamma>4 / 3$ [10]. In the long range case $\gamma \leq 1$, the ordinary wave operators are known not to exist in any reasonable sense [10], and should be replaced by modified wave operators including a suitable phase in their definition, as is the case for the linear Schrödinger equation. A well developed theory of long range scattering exists for the latter. See for instance [1] for a recent treatment and for an extensive bibliography. In contrast with that situation, only partial results are available for the Hartree equation. On the one hand, the existence of modified wave 
operators has been proved in the critical case $\gamma=1$ for small solutions [2]. On the other hand, it has been shown, first in the critical case $\gamma=1$ [6, 9] and then in the whole range $0<\gamma \leq 1$ [5, 7, 8] that the global solutions of the Hartree equation (1.1) (1.3) with small initial data exhibit an asymptotic behaviour as $t \rightarrow \pm \infty$ of the expected scattering type characterized by scattering states $u_{ \pm}$and including suitable phase factors that are typical of long range scattering. In particular, in the framework of scattering theory, the results of [5, 7, 8] are closely related to the property of asymptotic completeness for small data.

In a previous paper with the same title [4], hereafter referred to as I, we proved the existence of modified wave operators for the equation (1.1) (1.2), and we gave a description of the asymptotic behaviour in time of solutions in the ranges of those operators, with no size restriction on the data, in suitable spaces and for $\gamma$ in the range $1 / 2<\gamma<1$. The method is an extension of the energy method used in [5, 7, 8], and uses in particular the equations introduced in [7] to study the asymptotic behaviour of small solutions. The spaces of initial data, namely in the present case of asymptotic states, are Sobolev spaces of finite order similar to those used in [8]. The present paper is devoted to the extension of the previous results to the whole range $0<\gamma \leq 1$. The methods used here are natural extensions of those used in I. They require in particular the same restrictions on $\mu$ and $n$, in particular $\mu \leq n-2$ and $n \geq 3$. We refer to the introduction of I for a discussion of those conditions.

The construction of the modified wave operators is too complicated to allow for a more precise statement of results at this stage, and will be described in Section 2 below, which is a summary and continuation of Section 2 of I. That construction involves the study of the same auxiliary system of equations as in I, for a new function $w$ and a phase $\varphi$ instead of the original function $u$, and relies as a preliminary step on the construction of local wave operators in a neighborhood of infinity for that system. That step requires the definition of a modified asymptotic dynamics which is significantly more complicated than that used in I.

We now give a brief outline of the contents of this paper. A more detailed description of the technical parts will be given at the end of Section 2. After collecting some notation and preliminary estimates in Section 3 and recalling from I some preliminary results on the auxiliary system in Section 4, we define and study the asymptotic dynamics in Section 5. We then study the asymptotic behaviour of solutions for the auxiliary system in Section 6. In particular we 
essentially construct local wave operators at infinity for that system. We then come back from the auxiliary system to the original equation (1.1) for $u$ and construct the wave operators for the latter in Section 7, where the final result will be stated in Proposition 7.5.

We have tried to make this paper as self-contained as possible and at the same time to keep duplication with I to a minimum. Duplication occurs in the beginning of Section 3 and in Section 4 where we recall estimates and results from I. On the other hand, Sections 6 and 7 follow the same pattern as Sections 5, 6 and 7 of I, with the appropriate changes needed to handle the more general situation.

We conclude this section with some general notation which will be used freely throughout this paper. We denote by $\|\cdot\|_{r}$ the norm in $L^{r} \equiv L^{r}\left(I R^{n}\right)$. For any interval $I$ and any Banach space $X$, we denote by $\mathcal{C}(I, X)$ the space of strongly continuous functions from $I$ to $X$ and by $L^{\infty}(I, X)$ (resp. $\left.L_{l o c}^{\infty}(I, X)\right)$ the space of measurable essentially bounded (resp. locally essentially bounded) functions from $I$ to $X$. For real numbers $a$ and $b$, we use the notation $a \vee b=\operatorname{Max}(a, b), a \wedge b=\operatorname{Min}(a, b)$ and $[a]=$ integral part of $a$. In the estimates of solutions of the relevant equations, we shall use the letter $C$ to denote constants, possibly different from an estimate to the next, depending on various parameters such as $\gamma$, but not on the solutions themselves or on their initial data. Those constants will be bounded in $\gamma$ for $\gamma$ away from zero. We shall use the notation $A\left(a_{1}, a_{2}, \cdots\right)$ for estimating functions, also possibly different from an estimate to the next, depending in addition on suitable norms $a_{1}, a_{2}, \cdots$ of the solutions or of their initial data. Finally Item (p, q) of I will be referred to as Item (I. p. q). Additional notations will be given at the beginning of Section 3 .

In all this paper, we assume that $n \geq 3,0<\mu \leq n-2$ and $0<\gamma \leq 1$.

\section{Heuristics}

In this section, we discuss in heuristic terms the construction of the modified wave operators for the equation (1.1), as it will be performed in this paper. That construction is an extension of that performed in I in the special case $\gamma>1 / 2$, and we refer to Section I.2 for a more detailed introduction and for general background.

The problem that we want to address is that of classifying the possible asymptotic behaviours of the solutions of (1.1) by relating them to a set of model functions $\mathcal{V}=\left\{v=v\left(u_{+}\right)\right\}$ 
parametrized by some data $u_{+}$and with suitably chosen and preferably simple asymptotic behaviour in time. For each $v \in \mathcal{V}$, one tries to construct a solution $u$ of $(1.1)$ such that $u(t)$ behaves as $v(t)$ when $t \rightarrow \infty$ in a suitable sense. The map $\Omega: u_{+} \rightarrow u$ thereby obtained classifies the asymptotic behaviours of solutions of (1.1) and is a preliminary version of the wave operator for positive time. A similar question can be asked for $t \rightarrow-\infty$. From now on we restrict our attention to positive time.

In the short range case corresponding to $\gamma>1$ in (1.1), the previous scheme can be implemented by taking for $\mathcal{V}$ the set $\mathcal{V}=\left\{v=U(t) u_{+}\right\}$of solutions of the equation

$$
i \partial_{t} v+\frac{1}{2} \Delta v=0
$$

with $U(t)$ being the unitary group

$$
U(t)=\exp (i(t / 2) \Delta)
$$

The initial data $u_{+}$for $v$ is called the asymptotic state for $u$.

In the long range case corresponding to $\gamma \leq 1$ in (1.1) (1.2), the previous set is known to be inadequate and has to be replaced by a better set of model functions obtained by modifying the previous ones by a suitable phase. The modification that we use requires additional structure of $U(t)$. In fact $U(t)$ can be written as

$$
U(t)=M(t) D(t) F M(t)
$$

where $M(t)$ is the operator of multiplication by the function

$$
M(t)=\exp \left(i x^{2} / 2 t\right),
$$

$F$ is the Fourier transform and $D(t)$ is the dilation operator defined by

$$
(D(t) f)(x)=(i t)^{-n / 2} f(x / t)
$$

Let now $\varphi^{(0)}=\varphi^{(0)}(x, t)$ be a real function of space time and let $z^{(0)}(x, t)=\exp \left(-i \varphi^{(0)}(x, t)\right)$. We replace $v(t)=U(t) u_{+}$by the modified free evolution [12] [13]

$$
v(t)=M(t) D(t) z^{(0)}(t) w_{+}
$$


where $w_{+}=F u_{+}$. In order to allow for easy comparison of $u$ with $v$, it is then convenient to represent $u$ in terms of a phase factor $z(t)=\exp (-i \varphi(t))$ and of an amplitude $w(t)$ in such a way that asymptotically $\varphi(t)$ behaves as $\varphi^{(0)}(t)$ and $w(t)$ tends to $w_{+}$. This is done by writing $u$ in the form [7]

$$
u(t)=M(t) D(t) z(t) w(t) \equiv(\Lambda(w, \varphi))(t)
$$

In I, we introduced three possible modified free evolutions $v_{i}(t) i=1,2,3$ and correspondingly three parametrizations of $u(t)$ by $\left(w_{i}(t), \varphi_{i}(t)\right), i=1,2,3$. The choice (2.6) (2.7) corresponds to $i=2$. We shall work exclusively with that choice throughout this paper, and the subscript 2 is therefore consistently omitted. In I we used mostly the choice $i=3$ and dropped the subscript 3 , so that $(w, \varphi)$ in I means $\left(w_{3}, \varphi_{3}\right)$ as opposed to $\left(w_{2}, \varphi_{2}\right)$ in this paper. This should be kept in mind when comparing results from I and from this paper.

The construction of the wave operators for $u$ proceeds by first constructing the wave operators for the pair $(w, \varphi)$ and then recovering the wave operators for $u$ therefrom by the use of $(2.7)$. The evolution equation for $(w, \varphi)$ is obtained by substituting (2.7) into the equation (1.1). One obtains the equation

$$
\left(i \partial_{t}+\left(2 t^{2}\right)^{-1} \Delta-D^{*} \widetilde{g} D\right) z w=0
$$

for $z w$, with

$$
\widetilde{g} \equiv \widetilde{g}\left(|u|^{2}\right)=\widetilde{g}\left(|D w|^{2}\right)
$$

or equivalently, by expanding the derivatives in (2.8),

$$
\begin{gathered}
\left\{i \partial_{t}+\left(2 t^{2}\right)^{-1} \Delta-i\left(2 t^{2}\right)^{-1}(2 \nabla \varphi \cdot \nabla+(\Delta \varphi))\right\} w \\
+\left\{\partial_{t} \varphi-\left(2 t^{2}\right)^{-1}|\nabla \varphi|^{2}-D^{*} \widetilde{g} D\right\} w=0 .
\end{gathered}
$$

We are now in the situation of a gauge theory. The equation (2.8) or (2.10) is invariant under the gauge transformation $(w, \varphi) \rightarrow(w \exp (i \sigma), \varphi+\sigma)$, where $\sigma$ is an arbitrary function of space time, and the original gauge invariant equation is not sufficient to provide evolution equations for the two gauge dependent quantities $w$ and $\varphi$. At this point we arbitrarily add the Hamilton-Jacobi equation as a gauge condition. This yields a system of evolution equations for $(w, \varphi)$, namely 


$$
\left\{\begin{array}{l}
\partial_{t} w=i\left(2 t^{2}\right)^{-1} \Delta w+\left(2 t^{2}\right)^{-1}(2 \nabla \varphi \cdot \nabla+(\Delta \varphi)) w \\
\partial_{t} \varphi=\left(2 t^{2}\right)^{-1}|\nabla \varphi|^{2}+t^{-\gamma} g_{0}(w, w)
\end{array}\right.
$$

where we have defined

$$
g_{0}\left(w_{1}, w_{2}\right)=\lambda \operatorname{Re} \omega^{\mu-n} w_{1} \bar{w}_{2}
$$

and rewritten the nonlinear interaction term in (2.10) as

$$
D^{*} \widetilde{g}\left(|D w|^{2}\right) D=t^{-\gamma} g_{0}(w, w)
$$

The gauge freedom in (2.11) (2.12) is now reduced to that given by an arbitrary function of space only. It can be shown, actually it has been shown in I, that the Cauchy problem for the system (2.11) (2.12) is locally wellposed in a neighborhood of infinity in time. The solutions thereby obtained behave asymptotically as $w(t)=O(1)$ and $\varphi(t)=O\left(t^{1-\gamma}\right)$ as $t \rightarrow \infty$, a behaviour that is immediately seen to be compatible with (2.11) (2.12).

We next study the asymptotic behaviour of the solutions of the auxiliary system (2.11) (2.12) in more detail and try to construct wave operators for that system. For that purpose, we need to choose a set of model functions playing the role of $v$, in the spirit of (2.6). In the simple case $\gamma>1 / 2$ considered in I, that set of model functions was taken to consist of solutions of the system

$$
\left\{\begin{array}{l}
\partial_{t} w^{(0)}=0 \\
\partial_{t} \varphi^{(0)}=t^{-\gamma} g_{0}\left(w^{(0)}, w^{(0)}\right)
\end{array}\right.
$$

The general solution of $(2.14)$ is

$$
\left\{\begin{array}{l}
w^{(0)}(t)=w_{+} \\
\varphi^{(0)}(t)=\psi_{+}+\int_{1}^{t} d t_{1} t_{1}^{-\gamma} g_{0}\left(w_{+}, w_{+}\right) \equiv \psi_{+}+\varphi_{0}(t)
\end{array}\right.
$$

and leads to $(2.6)$ with $\varphi^{(0)}=\psi_{+}+\varphi_{0}$. The asymptotic states for $(w, \varphi)$ then consist of pairs $\left(w_{+}, \psi_{+}\right)$. The choice $(2.14)(2.15)$ is adequate for $\gamma>1 / 2$ because comparison of $(2.11)(2.12)$ with $(2.15)$ yields $\partial_{t}\left(\varphi-\varphi_{0}\right)=O\left(t^{-2 \gamma}\right)$ which is integrable at infinity for $\gamma>1 / 2$, thereby allowing for imposing an initial condition at $t=\infty$ for $\psi_{1}=\varphi-\varphi_{0}$. For $\gamma \leq 1 / 2$ however, the choice (2.14) (2.15) is not sufficient and one needs to construct more accurate asymptotic 
functions. There are several ways to do that. The one we choose can be motivated heuristically as follows. Let $p \geq 0$ be an integer. We write

$$
\left\{\begin{array}{l}
w=\sum_{0 \leq m \leq p} w_{m}+q_{p+1} \equiv W_{p}+q_{p+1} \\
\varphi=\sum_{0 \leq m \leq p} \varphi_{m}+\psi_{p+1} \equiv \phi_{p}+\psi_{p+1}
\end{array}\right.
$$

with the understanding that asymptotically in $t$

$$
\begin{gathered}
w_{m}(t)=O\left(t^{-m \gamma}\right) \quad, \quad q_{p+1}(t)=o\left(t^{-p \gamma}\right) \\
\varphi_{m}(t)=O\left(t^{1-(m+1) \gamma}\right) \quad, \quad \psi_{p+1}(t)=o\left(t^{1-(p+1) \gamma}\right)
\end{gathered}
$$

Substituting (2.16) (2.17) into (2.11) (2.12) and identifying the various powers of $t^{-\gamma}$ yields the following system of equations for $\left(w_{m}, \varphi_{m}\right)$ :

$$
\left\{\begin{array}{l}
\partial_{t} w_{m+1}=\left(2 t^{2}\right)^{-1} \sum_{0 \leq j \leq m}\left(2 \nabla \varphi_{j} \cdot \nabla+\left(\Delta \varphi_{j}\right)\right) w_{m-j} \\
\partial_{t} \varphi_{m+1}=\left(2 t^{2}\right)^{-1} \sum_{0 \leq j \leq m} \nabla \varphi_{j} \cdot \nabla \varphi_{m-j}+t^{-\gamma} \sum_{0 \leq j \leq m+1} g_{0}\left(w_{j}, w_{m+1-j}\right)
\end{array}\right.
$$

for $m+1 \geq 0$. Here it is understood that $w_{j}=0$ and $\varphi_{j}=0$ for $j<0$, so that the case $m=-1$ of $(2.20)(2.21)$ reduces to $(2.14)$ with $w^{(0)}=w_{0}$ and $\varphi^{(0)}=\varphi_{0}$. We supplement that system with the initial conditions

$$
\left\{\begin{array}{l}
w_{0}(\infty)=w_{+} \quad, \quad w_{m}(\infty)=0 \quad \text { for } m \geq 1 \\
\varphi_{m}(1)=0 \quad \text { for } 0 \leq m \leq p
\end{array}\right.
$$

The system (2.20) (2.21) with the initial conditions (2.22) (2.23) can be solved by successive integrations : knowing $\left(w_{j}, \varphi_{j}\right)$ for $0 \leq j \leq m$, one constructs successively $w_{m+1}$ by integrating (2.20) between $t$ and $\infty$, and then $\varphi_{m+1}$ by integrating (2.21) between 1 and $t$.

If $(p+1) \gamma<1$, that method of resolution reproduces the asymptotic behaviour in time (2.18) (2.19) which was used in the first place to provide a heuristic derivation of the system (2.20) (2.21). One can however consider that system and solve it by the same method for any integer $p$. If $(p+1) \gamma>1$, the asymptotic behaviour saturates at $w_{m}=O\left(t^{-1}\right)$ for $m \gamma>1$ and $\varphi_{m}=O(1)$ for $(m+1) \gamma>1$. If $\gamma^{-1}$ is an integer, $(m+1) \gamma=1$ for some $m$, then $\varphi_{m}(t)=$ $O(\log t)$ and $w_{m+1}=O\left(t^{-1} \log t\right)$.

We now argue that for sufficiently large $p, \phi_{p}$ is a sufficiently good approximation for $\varphi$ to ensure that $\psi_{p+1}$ has a limit as $t \rightarrow \infty$. In fact by comparing the system (2.20) (2.21) with (2.11) 
(2.12), one finds that $\partial_{t} \psi_{p+1}$ is of the same order in $t$ as $\partial_{t} \varphi_{p+1}$, namely $\partial_{t} \psi_{p+1}=O\left(t^{-(p+2) \gamma}\right)$, which is integrable at infinity for $(p+2) \gamma>1$. In this way every solution $(w, \varphi)$ of the system (2.11) (2.12) as obtained previously has asymptotic states consisting of $w_{+}=\lim _{t \rightarrow \infty} w(t)$ and $\psi_{+}=\lim _{t \rightarrow \infty} \psi_{p+1}(t)$

Conversely, under the condition $(p+2) \gamma>1$, we shall be able to solve the system $(2.11)$ (2.12) by looking for solutions in the form (2.16) (2.17) with the additional initial condition $\psi_{p+1}(\infty)=\psi_{+}$, thereby getting a solution which is asymptotic to $\left(W_{p}, \phi_{p}+\psi_{+}\right)$with

$$
w-W_{p}=O\left(t^{-(p+1) \gamma}\right) \quad, \quad \varphi-\phi_{p}-\psi_{+}=O\left(t^{1-(p+2) \gamma}\right)
$$

This allows to define a map $\Omega_{0}:\left(w_{+}, \psi_{+}\right) \rightarrow(w, \varphi)$ which is essentially the wave operator for $(w, \varphi)$.

It is an unfortunate feature of the methods used in this paper that both the construction of the asymptotic states $\left(w_{+}, \psi_{+}\right)$of a given solution $(w, \varphi)$ and the construction of $(w, \varphi)$ from given asymptotic states $\left(w_{+}, \psi_{+}\right)$suffer from a loss of regularity of roughly $p+1$ derivatives, which prevents the two constructions to be inverse of each other in a strict sense.

We next discuss the gauge covariance properties of $\Omega_{0}$. Two solutions $(w, \varphi)$ and $\left(w^{\prime}, \varphi^{\prime}\right)$ of the system (2.11) (2.12) will be said to be gauge equivalent if they give rise to the same $u$ through (2.7), namely if $w \exp (-i \varphi)=w^{\prime} \exp \left(-i \varphi^{\prime}\right)$. If $(w, \varphi)$ and $\left(w^{\prime}, \varphi^{\prime}\right)$ are two gauge equivalent solutions, one can show easily that the difference $\varphi_{-}=\varphi^{\prime}-\varphi$ has a limit $\sigma$ when $t \rightarrow \infty$ and that $w_{+}^{\prime}=w_{+} \exp (i \sigma)$. Under that condition, it turns out that the phases $\left\{\varphi_{j}\right\}$ and $\phi_{p}$ (but not the amplitudes) obtained by solving (2.20) (2.21) are gauge invariant, namely $\varphi_{m}=\varphi_{m}^{\prime}$ for $0 \leq m \leq p$ and therefore $\phi_{p}=\phi_{p}^{\prime}$, so that $\psi_{+}^{\prime}=\psi_{+}+\sigma$. It is then natural to define gauge equivalence of asymptotic states $\left(w_{+}, \psi_{+}\right)$and $\left(w_{+}^{\prime}, \psi_{+}^{\prime}\right)$ by the condition $w_{+} \exp \left(-i \psi_{+}\right)=$ $w_{+}^{\prime} \exp \left(-i \psi_{+}^{\prime}\right)$ and the previous result can be rephrased as the statement that gauge equivalent solutions of (2.11) (2.12) in $\mathcal{R}\left(\Omega_{0}\right)$ have gauge equivalent asymptotic states. Conversely, we are interested in showing that gauge equivalent asymptotic states have gauge equivalent images under $\Omega_{0}$. Here however we meet with a technical problem coming from the construction of $\Omega_{0}$ itself. For given $\left(w_{+}, \psi_{+}\right)$we construct $(w, \varphi)$ in practice as follows. We take a (large) finite time $t_{0}$ and we define a solution $\left(w_{t_{0}}, \varphi_{t_{0}}\right)$ of the system (2.11) (2.12) by imposing a suitable initial condition at $t_{0}$, depending on $\left(w_{+}, \psi_{+}\right)$, and using the known results for the Cauchy 
problem with finite initial time. We then let $t_{0}$ tend to infinity and obtain $(w, \varphi)$ as the limit of $\left(w_{t_{0}}, \varphi_{t_{0}}\right)$. The simplest way to prove the gauge equivalence of two solutions $(w, \varphi)$ and $\left(w^{\prime}, \varphi^{\prime}\right)$ obtained in this way from gauge equivalent $\left(w_{+}, \psi_{+}\right)$and $\left(w_{+}^{\prime}, \psi_{+}^{\prime}\right)$ consists in using an initial condition at $t_{0}$ which already ensures that $\left(w_{t_{0}}, \varphi_{t_{0}}\right)$ and $\left(w_{t_{0}}^{\prime}, \varphi_{t_{0}}^{\prime}\right)$ are gauge equivalent. Unfortunately the natural choice $\left(w_{t_{0}}\left(t_{0}\right), \varphi_{t_{0}}\left(t_{0}\right)\right)=\left(W_{p}\left(t_{0}\right), \phi_{p}\left(t_{0}\right)+\psi_{+}\right)$does not satisfy that requirement as soon as $p \geq 1$ because $\phi_{p}\left(t_{0}\right)$ is gauge invariant while $W_{p}\left(t_{0}\right) \exp \left(-\psi_{+}\right)$is not. In order to overcome that difficulty, we introduce a new amplitude $V$ and a new phase $\chi$ defined by solving the transport equations

$$
\left\{\begin{array}{l}
\partial_{t} V=\left(2 t^{2}\right)^{-1}\left(2 \nabla \phi_{p-1} \cdot \nabla+\left(\Delta \phi_{p-1}\right)\right) V \\
\partial_{t} \chi=t^{-2} \nabla \phi_{p-1} \cdot \nabla \chi
\end{array}\right.
$$

with initial condition

$$
V(\infty)=w_{+} \quad, \quad \chi(\infty)=\psi_{+}
$$

It follows from $(2.25)(2.26)$ that $V \exp (-i \chi)$ satisfies the same transport equation as $V$, now with gauge invariant initial condition $(V \exp (-i \chi))(\infty)=w_{+} \exp \left(-i \psi_{+}\right)$, and is therefore gauge invariant. Furthermore, $(V, \chi)$ is a sufficiently good approximation of $\left(W_{p}, \psi_{+}\right)$in the sense that

$$
V(t)-W_{p}(t)=O\left(t^{-(p+1) \gamma}\right) \quad, \quad \chi(t)-\psi_{+}=O\left(t^{-\gamma}\right)
$$

One then takes $\left(w_{t_{0}}\left(t_{0}\right), \varphi_{t_{0}}\left(t_{0}\right)\right)=\left(V\left(t_{0}\right), \phi_{p}\left(t_{0}\right)+\chi\left(t_{0}\right)\right)$ as an initial condition at time $t_{0}$, thereby ensuring that $\left(w_{t_{0}}, \varphi_{t_{0}}\right)$ and $\left(w_{t_{0}}^{\prime}, \varphi_{t_{0}}^{\prime}\right)$ are gauge equivalent. That equivalence is easily seen to be preserved in the limit $t_{0} \rightarrow \infty$. Furthermore, the estimates (2.28) ensure that the asymptotic properties (2.24) are preserved by the modified construction. As a consequence of the previous discussion, the map $\Omega_{0}$ is gauge covariant, namely induces an injective map of gauge equivalence classes of asymptotic states $\left(w_{+}, \psi_{+}\right)$to gauge equivalence classes of solutions $(w, \varphi)$ of the system $(2.11)(2.12)$.

The wave operator for $u$ is obtained from $\Omega_{0}$ just defined and from $\Lambda$ defined by (2.7). From the previous discussion it follows that the map $\Lambda \circ \Omega_{0}:\left(w_{+}, \psi_{+}\right) \rightarrow u$ is injective from gauge equivalence classes of asymptotic states $\left(w_{+}, \psi_{+}\right)$to solutions of (1.1). In order to define a wave operator for $u$ involving only the asymptotic state $u_{+}$but not an arbitrary phase $\psi_{+}$, we choose a representative in each equivalence class $\left(w_{+}, \psi_{+}\right)$, namely we define the wave operator for $u$ as the map $\Omega: u_{+} \rightarrow u=\left(\Lambda \circ \Omega_{0}\right)\left(F u_{+}, 0\right)$. Since each equivalence class of asymptotic states 
contains at most one element with $\psi_{+}=0$, the map $\Omega$ is again injective. We shall prove in addition that $\mathcal{R}(\Omega)=\mathcal{R}\left(\Lambda \circ \Omega_{0}\right)$ if $p \leq 2$. (This need not be the case if $p \geq 3$, because derivative losses in the construction generate a mismatch between the regularity properties required on $w_{+}$and $\psi_{+}$, so that gauge equivalence classes of asymptotic states need not contain an element with $\psi_{+}=0$ in that case).

The previous heuristic discussion was based in part on a number of asymptotic estimates in terms of negative powers of $t$. However if $\gamma^{-1}$ is an integer some of these estimates have to be replaced or supplemented by logarithms. In order to treat all values of $\gamma \in(0,1]$ in a unified way, we shall introduce a number of estimating functions of time defined by integral representations. Those functions are smooth in $\gamma$, in particular at integer values of $\gamma^{-1}$. They generate the logarithms automatically whenever needed, and they recombine nicely between themselves in the derivation of the main estimates. The simplest example thereof is $h_{0}(t)$ defined by (3.19) below.

In the same way as in I, the system (2.11) (2.12) can be rewritten as a system of equations for $w$ and for $s=\nabla \varphi$, from which $\varphi$ can then be recovered by (2.12), thereby leading to a slightly more general theory since the system for $(w, s)$ can be studied without even assuming that $s$ is a gradient. In I, we first studied the system for $(w, s)$ and then deduced therefrom the relevant results for $(w, \varphi)$. Here for simplicity we shall use exclusively the variables $(w, \varphi)$. The same remark applies to the system (2.20) (2.21).

We are now in a position to describe in more detail the contents of the technical parts of this paper, namely Sections 3-7. In Section 3, we introduce some notation, we define the relevant function spaces needed to study the system (2.11) (2.12), we recall from I a number of Sobolev and energy estimates, we then introduce the estimating functions of time mentioned above and we derive a number of estimates for them. In Section 4, we recall from I some preliminary results on the Cauchy problem for the auxiliary system (2.11) (2.12) and on the asymptotic behaviour of its solutions. In Section 5 we study the systems (2.20) (2.21) and (2.25) (2.26) defining the asymptotic dynamics. We first derive a number of properties and estimates for the solutions of the system (2.20) (2.21), defined inductively (Proposition 5.1). We then prove the existence and some properties of the solutions of the transport equations (2.25) and (2.26) in a slightly more general setting (Propositions 5.2 and 5.3 respectively). We finally specialize 
those results to the case at hand and compare $V$ with $W_{p}$ defined by (2.16) (Proposition 5.4). In Section 6 we study in detail the asymptotic behaviour in time of solutions of the auxiliary system (2.11) (2.12). We first derive asymptotic estimates on the approximation of the available solutions $(w, \varphi)$ of that system by the asymptotic functions $\left(W_{m}, \phi_{m}\right)$ defined by $(2.16)(2.17)$, and in particular we complete the proof of existence of asymptotic states for those solutions (Proposition 6.1). We then turn to the construction of local wave operators at infinity. For a given solution $(V, \chi)$ of the system (2.25) (2.26) and a given (large) $t_{0}$, we construct a solution $\left(w_{t_{0}}, \varphi_{t_{0}}\right)$ of the system (2.11) (2.12) which coincides with $\left(V, \phi_{p}+\chi\right)$ at $t_{0}$ and we estimate it uniformly in $t_{0}$ (Proposition 6.2). We then prove that when $t_{0} \rightarrow \infty,\left(w_{t_{0}}, \varphi_{t_{0}}\right)$ has a limit $(w, \varphi)$ which is asymptotic both to $\left(V, \phi_{p}+\chi\right)$ and to $\left(W_{p}, \phi_{p}+\psi_{+}\right)$(Proposition 6.3). Finally in Section 7, we exploit the results of Section 6 to construct the wave operators for the equation (1.1) and to describe the asymptotic behaviour of solutions in their range. We first prove that the local wave operator at infinity for the system (2.11) (2.12) defined through Proposition 6.3 in Definition 7.1 is gauge covariant in the sense of Definitions 7.2 and 7.3 in the best form that can be expected with the available regularity (Propositions 7.2 and 7.3). With the help of some information on the Cauchy problem for (1.1) at finite time (Proposition 7.1), we then define the wave operator $\Omega: u_{+} \rightarrow u$ (Definition 7.4), we prove that it is injective and under suitable restrictions, that it has the expected range (Proposition 7.4). We then collect all the available information on $\Omega$ and on solutions of (1.1) in its range in Proposition 7.5, which contains the main results of this paper.

\section{Notation and preliminary estimates}

In this section, we define the function spaces where we shall study the auxiliary system (2.11) (2.12) and we recall from I a number of Sobolev and energy type estimates which hold in those spaces. We then introduce a number of estimating functions of time and we derive a number of relations and estimates for them.

We shall use Sobolev spaces of integer order $H_{r}^{k}$ defined for $1 \leq r \leq \infty$ by

$$
H_{r}^{k}=\left\{u:\left\|u ; H_{r}^{k}\right\| \equiv \sum_{0 \leq j \leq k}\left\|\partial^{j} u\right\|_{r}<\infty\right\}
$$


and the associated homogeneous spaces $\dot{H}_{r}^{k}$ with norm

$$
\left\|u ; \dot{H}_{r}^{k}\right\|=\left\|\partial^{k} u\right\|_{r}
$$

where

$$
\left\|\partial^{j} u\right\|_{r}=\sum_{\alpha:|\alpha|=j}\left\|\partial^{\alpha} u\right\|_{r}
$$

The subscript $r$ will be omitted if $r=2$.

Let $\ell_{0}=[n / 2]$ and define $r_{0}$ by $\delta\left(r_{0}\right)=\ell_{0}$ so that $r_{0}=2 n$ for odd $n$ and $r_{0}=\infty$ for even $n$. Let $k$ and $\ell$ be nonnegative integers with $\ell \geq \ell_{0}-1$. We shall look for $w$ as a complex valued function in spaces $L_{l o c}^{\infty}\left(I, H^{k}\right)$ or $\mathcal{C}\left(I, H^{k}\right)$ and for $\varphi$ as a real valued function in spaces $L_{\text {loc }}^{\infty}\left(I, Y^{\ell}\right)$ or $\mathcal{C}\left(I, Y^{\ell}\right)$ where

$$
Y^{\ell}=L^{\infty} \cap \dot{H}_{r_{0}}^{1} \cap \dot{H}^{\ell_{0}+1} \cap \dot{H}^{\ell+2}
$$

The spaces $Y^{\ell}$ are easily seen to be duals of Banach spaces and satisfy the embedding $Y^{\ell^{\prime}} \subset Y^{\ell}$ for $\ell^{\prime} \geq \ell$. We shall use systematically the notation

$$
|w|_{k}=\left\|w ; H^{k}\right\| \quad, \quad|\varphi|_{\ell}=\left\|\varphi ; Y^{\ell}\right\|
$$

and the meaning of the symbol $|a|_{b}$ will be made unambiguous by the fact that the pair $(a, b)$ contains either the pair $(w, k)$ or the pair $(\varphi, \ell)$. Note that the second notation in $(3.2)$ is different from, although closely related to, the similar notation in I which was used for $s=\nabla \varphi$.

We recall the following result from I (see Lemma I.3.5).

Lemma 3.1. Let $\varphi$ be a real function with $\nabla \varphi \in L^{\infty} \cap \dot{H}^{\ell}$ for some $\ell>n / 2$ and let $k \leq \ell+1$. Then the following estimate holds :

$$
|\exp (-i \varphi) w|_{k} \leq C\left(1+\left\|\nabla \varphi ; L^{\infty} \cap \dot{H}^{\ell}\right\|\right)^{k}|w|_{k}
$$

Let in addition $\varphi \in L^{\infty}$. Then the following estimate holds :

$$
|(\exp (-i \varphi)-1) w|_{k} \leq C\left(\|\varphi\|_{\infty}+\left\|\nabla \varphi ; L^{\infty} \cap \dot{H}^{\ell}\right\|\left(1+\left\|\nabla \varphi ; L^{\infty} \cap \dot{H}^{\ell}\right\|\right)^{k-1}\right)|w|_{k} .
$$

In order to state the estimates that are relevant for the study of the system (2.11) (2.12), it is useful to give the following definition (see Definition I.3.1). 
Definition 3.1. Let $0<\mu \leq n-2$. A pair of nonnegative integers $(k, \ell)$ will be called admissible if it satisfies $k \leq \ell, \ell>n / 2$ and

$$
\ell+2+\mu \leq(n / 2+2 k) \wedge(n+k)
$$

and in addition $k>n / 2$ if $\ell+2+\mu=n+k$ and

$$
n / 2+3+\mu<(n / 2+2 k) \wedge(n+k)
$$

if $n$ is even.

For $\mu=n-2$, admissible pairs are pairs $(k, \ell)$ such that $k=\ell>n / 2$. If $(k, \ell)$ is admissible, so is $(k+j, \ell+j)$ for any positive integer $j$. Admissible pairs always have $k \geq 2$. For $n=3$, $\mu=1$, the pair $(2,2)$ is admissible.

The following Sobolev like inequalities will be essential to study the system (2.11) (2.12).

Lemma 3.2. Let $\ell>n / 2$ and $k \leq \ell$. Then the following estimates hold:

$$
\begin{gathered}
|(2 \nabla \varphi \cdot \nabla+(\Delta \varphi)) w|_{k-1} \leq C|\varphi|_{\ell-1}|w|_{k}, \\
\left|\nabla \varphi_{1} \cdot \nabla \varphi_{2}\right|_{\ell-1} \leq C\left|\varphi_{1}\right|_{\ell}\left|\varphi_{2}\right|_{\ell} .
\end{gathered}
$$

Assume in addition that $(k, \ell)$ is admissible. Let $g_{0}$ be defined by (2.13). Then

$$
\begin{gathered}
\left|g_{0}\left(w_{1} w_{2}\right)\right|_{\ell} \leq C\left|w_{1}\right|_{k}\left|w_{2}\right|_{k}, \\
\left|g_{0}\left(w_{1} w_{2}\right)\right|_{\ell-1} \leq C\left|w_{1}\right|_{k}\left|w_{2}\right|_{k-1} .
\end{gathered}
$$

Sketch of proof. (3.6) follows from Lemma I.3.4 by the same estimates as in Lemma I.3.9. The estimate (3.7) essentially follows from Lemma I.3.3. The estimates (3.8) and (3.9) follow from Corollary I.3.1.

In addition to the previous estimates, we shall need energy type estimates for solutions of the following transport equations

$$
\partial_{t} w=\left(2 t^{2}\right)^{-1}\{i \theta \Delta w+(2 \nabla \phi \cdot \nabla+(\Delta \phi)) w\}+R_{1}
$$




$$
\partial_{t} \varphi=\left(2 t^{2}\right)^{-1}\left\{\theta|\nabla \varphi|^{2}+2 \nabla \phi \cdot \nabla \varphi\right\}+R_{2}
$$

where $\theta$ is a real constant and $\phi, R_{1}, R_{2}$ are given functions of space time. Those estimates will be stated in differential form for brevity, although they should be understood in integrated from. They hold for functions that are sufficiently regular in time, for instance locally bounded in the relevant norms.

Lemma 3.3. Let $\ell>n / 2$ and $k \leq \ell$.

(1) Let $w$ satisfy (3.10). Then the following estimate holds :

$$
\left.\left.\left|\partial_{t}\right| w\right|_{k}\left|\leq C t^{-2}\right| \phi\right|_{\ell}|w|_{k}+\left|R_{1}\right|_{k}
$$

(2) Let $\varphi$ satisfy (3.11). Then the following estimates hold:

$$
\begin{gathered}
\left.\left.\left|\partial_{t}\right| \varphi\right|_{\ell}\left|\leq C t^{-2}\right| \varphi\right|_{\ell}\left(|\theta||\varphi|_{\ell}+|\phi|_{\ell+1}\right)+\left|R_{2}\right|_{\ell} \\
\left.\left.\left|\partial_{t}\right| \varphi\right|_{\ell-1}\left|\leq C t^{-2}\right| \varphi\right|_{\ell-1}\left(|\theta||\varphi|_{\ell}+|\phi|_{\ell}\right)+\left|R_{2}\right|_{\ell-1}
\end{gathered}
$$

\section{Sketch of proof.}

(3.12) follows from Lemmas I.3.2 and I.3.4 by the same estimates as in Lemma I.3.7.

(3.13) and (3.14) follow from Lemmas I.3.2 and I.3.3 by the same estimates as in Lemmas I.3.7 and I.3.9.

Lemma 3.4. Let $\ell>n / 2$ and $k \leq \ell$. Let $w$ and $\varphi$ satisfy (3.10) and (3.11) respectively, with $\theta=0, R_{1}=0$ and $R_{2}=0$. Then the following estimates hold:

$$
\begin{aligned}
& \left.\left|\partial_{t}\right| w\right|_{k+1} \mid \leq C t^{-2}\left(|\phi|_{\ell}|w|_{k+1}+|\phi|_{\ell+1}|w|_{k}\right) \\
& \left.\left|\partial_{t}\right| \varphi\right|_{\ell+1} \mid \leq C t^{-2}\left(|\phi|_{\ell}|\varphi|_{\ell+1}+|\phi|_{\ell+2}|\varphi|_{\ell}\right)
\end{aligned}
$$

\section{Sketch of proof.}

(3.15) and (3.16) follow from Lemmas I.3.2, I.3.3 and I.3.4 by the same estimates as in Lemma I.3.8. 
Lemma 3.5. Let $\ell>n / 2$ and $k \leq \ell$. Let $w_{1}, w_{2}$ and $\varphi_{1}, \varphi_{2}$ satisfy (3.10) and (3.11) with $\phi=\phi_{1}$ and $\phi=\phi_{2}$ respectively, and with $\theta=0, R_{1}=0$ and $R_{2}=0$. Let $w_{-}=w_{1}-w_{2}$, $\varphi_{-}=\varphi_{1}-\varphi_{2}$ and $\phi_{-}=\phi_{1}-\phi_{2}$. Then the following estimates hold:

$$
\begin{gathered}
\left.\left|\partial_{t}\right| w_{-}\right|_{k} \mid \leq C t^{-2}\left(\left|\phi_{2}\right|_{\ell}\left|w_{-}\right|_{k}+\left|\phi_{-}\right|_{\ell}\left|w_{1}\right|_{k}+\left\|\nabla \phi_{-}\right\|_{\infty}\left|w_{1}\right|_{k+1}\right) \\
\left|\partial_{t}\right| \varphi_{-}|\ell| \leq C t^{-2}\left(\left|\phi_{2}\right|_{\ell+1}\left|\varphi_{-}\right|_{\ell}+\left|\phi_{-}\right|_{\ell+1}\left|\varphi_{1}\right|_{\ell}+\left\|\nabla \phi_{-}\right\|_{\infty}\left|\varphi_{1}\right|_{\ell+1}\right)
\end{gathered}
$$

\section{Sketch of proof.}

(3.17) and (3.18) follow from Lemmas I.3.2, I.3.3 and I.3.4 by the same estimates as in Lemma I.3.10.

We now introduce a number of estimating functions of time and derive a number of estimates and relations for them. We start with

$$
h_{0}(t)=\int_{1}^{t} d t_{1} t_{1}^{-\gamma}
$$

so that

$$
h_{0}(t)= \begin{cases}(1-\gamma)^{-1}\left(t^{1-\gamma}-1\right) & \text { for } \gamma \neq 1 \\ \log t & \text { for } \gamma=1 .\end{cases}
$$

The basic building block for the subsequent functions is the function $h$ defined by

$$
h(t)=\int_{1}^{\infty} d t_{1} t_{1}^{-\gamma}\left(t \vee t_{1}\right)^{-1}
$$

which can also be written as

$$
h(t)=t^{-1} h_{0}(t)+\gamma^{-1} t^{-\gamma}=\int_{t}^{\infty} d t_{1} t_{1}^{-2} h_{0}\left(t_{1}\right)
$$

and is explicitly computed as

$$
h(t)= \begin{cases}\gamma^{-1}(1-\gamma)^{-1}\left(t^{-\gamma}-\gamma t^{-1}\right) & \text { for } \gamma \neq 1 \\ t^{-1}(1+\log t) & \text { for } \gamma=1 .\end{cases}
$$

It follows from (3.21) that $t h(t)$ is increasing in $t$ and from (3.20) (3.23) that $t h(t) h_{0}(t)^{-1}$ is decreasing in $t$. The function $h$ satisfies the estimates

$$
\gamma^{-1}\left(t^{-\gamma} \vee t^{-1}\right) \leq h(t) \leq|1-\gamma|^{-1}\left(\gamma^{-1} t^{-\gamma} \vee t^{-1}\right)
$$


The first inequality in (3.24) follows in part from (3.22) and in part from the monotony of $t h(t)$, while the second inequality follows from (3.23) and holds only for $\gamma \neq 1$.

In some cases where we shall need to indicate the dependence of $h_{0}$ and $h$ on $\gamma$, we shall write $h_{0}(\gamma, t)$ and $h(\gamma, t)$ for $h_{0}(t)$ and $h(t)$.

We next define for any $m \geq 0$

$$
\begin{gathered}
N_{m}(t)=\int_{1}^{t} d t_{1} t_{1}^{-\gamma} h^{m}\left(t_{1}\right) \\
Q_{m}(t)=\int_{1}^{\infty} d t_{1} t_{1}^{-\gamma}\left(t \vee t_{1}\right)^{-1} h^{m}\left(t_{1}\right),
\end{gathered}
$$

so that $N_{0}=h_{0}$ and $Q_{0}=h$. Those functions are smooth in $\gamma$. Clearly $N_{m}$ is increasing and $Q_{m}$ is decreasing in $t$, while $t Q_{m}(t)$ is increasing in $t$, so that $Q_{m}(t) \geq Q_{m}(1) t^{-1}$. From the fact that $h$ is decreasing, it follows that

$$
\begin{gathered}
N_{i+j}(t) \leq \gamma^{-j} N_{i}(t) \leq \gamma^{-(i+j)} h_{0}(t) \\
Q_{i+j}(t) \leq \gamma^{-j} Q_{i}(t) \leq \gamma^{-(i+j)} h(t)
\end{gathered}
$$

for all $i \geq 0, j \geq 0$. It follows from (3.24) that $N_{m}$ and $Q_{m}$ satisfy the lower and upper bounds

$$
\begin{gathered}
\gamma^{-m} h_{0}((m+1) \gamma, t) \leq N_{m}(t) \leq(1-\gamma)^{-m} \gamma^{-m} h_{0}((m+1) \gamma, t) \\
\gamma^{-m} h((m+1) \gamma, t) \leq Q_{m}(t) \leq(1-\gamma)^{-m} \gamma^{-m} h((m+1) \gamma, t)
\end{gathered}
$$

where the lower bounds hold for all $\gamma>0$ and the upper bounds for $0<\gamma<1$ if $m \geq 1$. From (3.20) and (3.23), it follows that $N_{m}(t)$ and $Q_{m}(t)$ behave as $t^{1-(m+1) \gamma}$ and $t^{-(m+1) \gamma}$ respectively as $t \rightarrow \infty$ if $(m+1) \gamma<1$. If $(m+1) \gamma=1, N_{m}(t)$ and $Q_{m}(t)$ produce logarithms and behave as $\log t$ and $t^{-1} \log t$ respectively as $t \rightarrow \infty$. If $(m+1) \gamma>1, N_{m}(t)$ and $Q_{m}(t)$ saturate respectively as Constant and $t^{-1}$ when $t \rightarrow \infty$. For $m \geq 1$, the upper bounds in (3.29) and (3.30) blow up when $\gamma$ tends to one, but the same conclusions still hold.

For $(m+2) \gamma>1$, we finally define

$$
\begin{gathered}
P_{m}(t)=\int_{1}^{\infty} d t_{1} t_{1}^{-\gamma} h\left(t \vee t_{1}\right) h^{m}\left(t_{1}\right) \\
R_{m}(t)=\int_{t}^{\infty} d t_{1} t_{1}^{-2} P_{m}\left(t_{1}\right)
\end{gathered}
$$

In particular $P_{0}$ is explicitly computed as

$$
P_{0}(t)=h_{0}(t)\left(h(t)+t^{-1} t^{-\gamma}\right)+2 \gamma^{-1}(2 \gamma-1)^{-1} t^{1-2 \gamma}
$$


Clearly $P_{m}(t)$ and $R_{m}(t)$ are decreasing in $t$, while $P_{m}(t) h(t)^{-1}$ is increasing in $t$, so that $P_{m}(t) \geq P_{m}(1) \gamma h(t)$. It follows from (3.24) that $P_{m}$ satisfies the lower and upper bounds

$$
P_{m}(t)\left\{\begin{array}{l}
\geq 1 \\
\leq(1-\gamma)^{-(m+1)}
\end{array}\right\} \gamma^{-(m+1)}\left(t^{-\gamma} h_{0}((m+1) \gamma, t)+((m+2) \gamma-1)^{-1} t^{1-(m+2) \gamma}\right)
$$

From (3.20) it follows that $P_{m}(t)$ behaves as $t^{1-(m+2) \gamma}$ as $t \rightarrow \infty$ if $(m+1) \gamma<1$. If $(m+1) \gamma=1$, $P_{m}(t)$ behaves as $t^{-\gamma} \log t$. If $(m+1) \gamma>1, P_{m}(t)$ saturates at $t^{-\gamma}$ as long as $\gamma<1$.

We now collect a number of relations and estimates satisfied by the previous estimating functions.

Lemma 3.6. Let $i, j$ and $m$ be nonnegative integers. Let $1 \leq a \leq b$ and $t \geq 1$. Then the following identities and estimates hold:

$$
\begin{aligned}
& \int_{t}^{\infty} d t_{1} t_{1}^{-2} N_{m}\left(t_{1}\right)=Q_{m}(t) \\
& \int_{1}^{t} d t_{1} t_{1}^{-2} h_{0}\left(t_{1}\right) N_{m}\left(t_{1}\right)=N_{m+1}(t)-h(t) N_{m}(t) \leq N_{m+1}(t) \\
& \int_{t}^{\infty} d t_{1} t_{1}^{-2} h_{0}\left(t_{1}\right) N_{m}\left(t_{1}\right)=P_{m}(t) \quad \text { for }(m+2) \gamma>1 \\
& \int_{a}^{b} d t t^{-2} N_{i}(t) N_{j}(t) \leq \int_{a}^{b} d t t^{-2} h_{0}(t) N_{i+j}(t) \\
& \int_{a}^{b} d t t^{-2} N_{i}(t) Q_{j}(t) \leq \int_{a}^{b} d t t^{-2} h(t) N_{i+j}(t) \leq \int_{a}^{b} d t t^{-2} N_{i+j+1}(t) \\
& \int_{a}^{b} d t t^{-\gamma} Q_{i}(t) Q_{j}(t) \leq \int_{a}^{b} d t t^{-\gamma} h(t) Q_{i+j}(t) \\
& \int_{t}^{\infty} d t_{1} t_{1}^{-\gamma} h\left(t_{1}\right) Q_{m-1}\left(t_{1}\right) \leq \int_{t}^{\infty} d t_{1} t_{1}^{-\gamma} Q_{m}\left(t_{1}\right) \quad \text { for } m \geq 1,(m+2) \gamma>1 \text {. } \\
& \int_{t}^{\infty} d t_{1} t_{1}^{-\gamma} Q_{m}\left(t_{1}\right) \leq P_{m}(t) \quad \text { for }(m+2) \gamma>1 \text {. } \\
& \int_{1}^{t} d t_{1} t_{1}^{-\gamma} h\left(t_{1}\right) Q_{m-1}\left(t_{1}\right) \leq N_{m+1}(t) \\
& \int_{1}^{t} d t_{1} t_{1}^{-\gamma} Q_{m}\left(t_{1}\right) \leq N_{m+1}(t) \\
& \int_{a}^{b} d t t^{-\gamma} Q_{m}(t) \leq Q_{m}(a)\left(h_{0}(b)-h_{0}(a)\right) \\
& \int_{a}^{b} d t t^{-\gamma} h(t) Q_{m-1}(t) \leq 2 Q_{m}(a)\left(h_{0}(b)-h_{0}(a)\right) \\
& R_{m}(t) \leq C_{m} h(t) Q_{m}(t) \quad \text { for }(m+2) \gamma>1
\end{aligned}
$$


where

$$
C_{m}=(2 m+3) \gamma((m+2) \gamma-1)^{-1}
$$

\section{Proof.}

(3.35). By the definition of $N_{m}$ and $Q_{m}$

$$
\begin{gathered}
\int_{t}^{\infty} d t_{1} t_{1}^{-2} N_{m}\left(t_{1}\right)=\int_{t}^{\infty} d t_{1} t_{1}^{-2} \int_{1}^{t_{1}} d t_{2} t_{2}^{-\gamma} h^{m}\left(t_{2}\right) \\
=\int_{1}^{\infty} d t_{2} t_{2}^{-\gamma} h^{m}\left(t_{2}\right) \int_{t \vee t_{2}}^{\infty} d t_{1} t_{1}^{-2}=\int_{1}^{\infty} d t_{2} t_{2}^{-\gamma}\left(t \vee t_{2}\right)^{-1} h^{m}\left(t_{2}\right)=Q_{m}(t)
\end{gathered}
$$

(3.36). By the definition of $N_{m}$ and integration by parts

$$
\begin{gathered}
\int_{1}^{t} d t_{1} t_{1}^{-2} h_{0}\left(t_{1}\right) N_{m}\left(t_{1}\right)=-\int_{1}^{t} d t_{1} h^{\prime}\left(t_{1}\right) N_{m}\left(t_{1}\right) \\
=-h(t) N_{m}(t)+\int_{1}^{t} h(t) N_{m}^{\prime}(t)=N_{m+1}(t)-h(t) N_{m}(t) .
\end{gathered}
$$

(3.37). By the definitions of $N_{m}$ and $P_{m}$ and integration by parts

$$
\begin{gathered}
\int_{t}^{\infty} d t_{1} t_{1}^{-2} h_{0}\left(t_{1}\right) N_{m}\left(t_{1}\right)=h(t) N_{m}(t)+\int_{t}^{\infty} d t_{1} t_{1}^{-\gamma} h^{m+1}\left(t_{1}\right) \\
=\int_{1}^{\infty} d t_{1} t_{1}^{-\gamma} h\left(t \vee t_{1}\right) h^{m}\left(t_{1}\right)=P_{m}(t) .
\end{gathered}
$$

(3.38). By the definition of $N_{m}$

$$
\int_{a}^{b} d t t^{-2} N_{i}(t) N_{j}(t)=\int_{a}^{b} d t t^{-2} \int_{1}^{t} d t_{1} t_{1}^{-\gamma} h^{i}\left(t_{1}\right) \int_{1}^{t} d t_{2} t_{2}^{-\gamma} h^{j}\left(t_{2}\right)
$$

For fixed $i+j$, the last integral is logarithmically convex in $i$ (or $j$ ) and therefore estimated by the maximum of its values for $i=0$ and $j=0$, which are equal by symmetry and equal to the RHS of (3.38).

(3.39). By the definition of $N_{m}$ and $Q_{m}$

$$
\int_{a}^{b} d t t^{-2} N_{i}(t) Q_{j}(t)=\int_{a}^{b} d t t^{-2} \int_{1}^{t} d t_{1} t_{1}^{-\gamma} h^{i}\left(t_{1}\right) \int_{1}^{\infty} d t_{2} t_{2}^{-\gamma}\left(t \vee t_{2}\right)^{-1} h^{j}\left(t_{2}\right)
$$

We split the integral over $t_{2}$ into the subregions $t_{2} \leq t$ and $t_{2} \geq t$. In the region $t_{2} \leq t$, by logarithmic convexity and symmetry, we estimate the integral by replacing $h^{i}\left(t_{1}\right) h^{j}\left(t_{2}\right)$ by $h^{i+j}\left(t_{1}\right)$. In the region $t_{2} \geq t$, we make the same replacement because $t_{2} \geq t \geq t_{1}$ and $h$ is decreasing in $t$. We obtain

$$
\cdots \leq \int_{a}^{b} d t t^{-2} \int_{1}^{t} d t_{1} t_{1}^{-\gamma} h^{i+j}\left(t_{1}\right) h(t)=\int_{a}^{b} d t t^{-2} h(t) N_{i+j}(t)
$$


which yields the first inequality in (3.39). Using in addition the fact that $h(t) \leq h\left(t_{1}\right)$ for $t_{1} \leq t$ yields the second inequality.

(3.40). By the definition of $Q_{m}$, the LHS of (3.40) is logarithmically convex in $i$ or $j$ for fixed $i+j$, and symmetric in $i$ and $j$, and is therefore estimated by its end point values, namely with $i, j$ replaced by 0 and $i+j$.

(3.41) and (3.42). By the definition of $Q_{m}$

$$
\int_{t}^{\infty} d t_{1} t_{1}^{-\gamma} h\left(t_{1}\right) Q_{m-1}\left(t_{1}\right)=\int_{t}^{\infty} d t_{1} t_{1}^{-\gamma} h\left(t_{1}\right) \int_{1}^{\infty} d t_{2} t_{2}^{-\gamma}\left(t_{1} \vee t_{2}\right)^{-1} h^{m-1}\left(t_{2}\right)
$$

We estimate the last integral by replacing $h\left(t_{1}\right)$ by $h\left(t_{2}\right)$, by logarithmic convexity and symmetry in the region $t_{2} \geq t$ and by monotony of $h$ in the region $t_{2} \leq t\left(\leq t_{1}\right)$, thereby continuing (3.48) by

$$
\cdots \leq \int_{t}^{\infty} d t_{1} t_{1}^{-\gamma} \int_{1}^{\infty} d t_{2} t_{2}^{-\gamma}\left(t_{1} \vee t_{2}\right)^{-1} h^{m}\left(t_{2}\right)
$$

which is the RHS of (3.41) and the LHS of (3.42),

$$
\cdots=\int_{1}^{\infty} d t_{2} t_{2}^{-\gamma} h^{m}\left(t_{2}\right) \int_{t}^{\infty} d t_{1} t_{1}^{-\gamma}\left(t_{1} \vee t_{2}\right)^{-1}
$$

We estimate the last integral by $h\left(t \vee t_{2}\right)$ by first replacing $t_{1} \vee t_{2}$ by $t_{1} \vee t \vee t_{2}$, since $t_{1} \geq t$, and then extending the integration over $t_{1}$ to $[1, \infty)$, thereby obtaining

$$
\cdots \leq \int_{1}^{\infty} d t_{2} t_{2}^{-\gamma} h^{m}\left(t_{2}\right) h\left(t \vee t_{2}\right)=P_{m}(t)
$$

(3.43) and (3.44). By the definition of $Q_{m}$

$$
\begin{gathered}
\int_{1}^{t} d t_{1} t_{1}^{-\gamma}\left(h\left(t_{1}\right) Q_{m-1}\left(t_{1}\right) \text { or } Q_{m}\left(t_{1}\right)\right) \\
=\int_{1}^{t} d t_{1} t_{1}^{-\gamma} \int_{1}^{\infty} d t_{2} t_{2}^{-\gamma}\left(t_{1} \vee t_{2}\right)^{-1}\left(h\left(t_{1}\right) h^{m-1}\left(t_{2}\right) \text { or } h^{m}\left(t_{2}\right)\right) \\
\leq \int_{1}^{t} d t_{1} t_{1}^{-\gamma} h^{m}\left(t_{1}\right) \int_{1}^{\infty} d t_{2} t_{2}^{-\gamma}\left(t_{1} \vee t_{2}\right)^{-1}=N_{m+1}(t)
\end{gathered}
$$

by logarithmic convexity and symmetry in the region $t_{2} \leq t$ and by monotony of $h$ in the region $t_{2} \geq t\left(\geq t_{1}\right)$

(3.45) follows immediately from the fact that $Q_{m}$ is decreasing in $t$. 
(3.46). We first prove that

$$
h(t) Q_{m-1}(t) \leq 2 Q_{m}(t)
$$

In fact

$$
\begin{aligned}
& Q_{m-1}(t) h(t)=\int_{1}^{\infty} d t_{1} t_{1}^{-\gamma}\left(t \vee t_{1}\right)^{-1} \int_{1}^{\infty} d t_{2} t_{2}^{-\gamma}\left(t \vee t_{2}\right)^{-1} h^{m-1}\left(t_{1}\right) \\
& =\int_{1}^{\infty} d t_{1} t_{1}^{-\gamma}\left(t \vee t_{1}\right)^{-1} \int_{t_{1}}^{\infty} d t_{2} t_{2}^{-\gamma}\left(t \vee t_{1}\right)^{-1}\left(h^{m-1}\left(t_{1}\right)+h^{m-1}\left(t_{2}\right)\right) \\
& \leq 2 \int_{1}^{\infty} d t_{1} t_{1}^{-\gamma}\left(t \vee t_{1}\right)^{-1} \int_{t_{1}}^{\infty} d t_{2} t_{2}^{-\gamma}\left(t \vee t_{1} \vee t_{2}\right)^{-1} h^{m-1}\left(t_{1}\right) \leq 2 Q_{m}(t)
\end{aligned}
$$

since $h$ is decreasing in $t$ and

$$
\int_{t_{1}}^{\infty} d t_{2} t_{2}^{-\gamma}\left(t \vee t_{1} \vee t_{2}\right)^{-1} \leq h\left(t \vee t_{1}\right) \leq h\left(t_{1}\right)
$$

Now (3.46) follows from (3.49) and (3.45).

(3.47). We first define for future use

$$
\begin{gathered}
Q_{m}=Q_{m}^{-}+Q_{m}^{+}=t^{-1} \int_{1}^{t} d t_{1} t_{1}^{-\gamma} h^{m}\left(t_{1}\right)+\int_{t}^{\infty} d t_{1} t_{1}^{-1-\gamma} h^{m}\left(t_{1}\right) \\
P_{m}=P_{m}^{-}+P_{m}^{+}=h(t) \int_{1}^{t} d t_{1} t_{1}^{-\gamma} h^{m}\left(t_{1}\right)+\int_{t}^{\infty} d t_{1} t_{1}^{-\gamma} h^{m+1}\left(t_{1}\right) \\
R_{m}=R_{m}^{-}+R_{m}^{+}=\int_{t}^{\infty} d t_{1} t_{1}^{-2} P_{m}^{-}\left(t_{1}\right)+\int_{t}^{\infty} d t_{1} t_{1}^{-2} P_{m}^{+}\left(t_{1}\right)
\end{gathered}
$$

and we estimate $R_{m}^{-}$and $R_{m}^{+}$separately. We first estimate

$$
\begin{gathered}
R_{m}^{-}=\int_{t}^{\infty} d t_{1} t_{1}^{-2} h\left(t_{1}\right) \int_{1}^{t_{1}} d t_{2} t_{2}^{-\gamma} h^{m}\left(t_{2}\right) \\
\leq h(t) \int_{1}^{\infty} d t_{2} t_{2}^{-\gamma}\left(t \vee t_{2}\right)^{-1} h^{m}\left(t_{2}\right)=h(t) Q_{m}(t)
\end{gathered}
$$

by the monotony of $h$ and after performing the integral over $t_{1}$. We next use the differential equation

$$
\gamma h+t h^{\prime}=t^{-1}
$$

satisfied by $h$ to rewrite $P_{m}^{+}$as follows

$$
\gamma P_{m}^{+}(t)=-\int_{t}^{\infty} d t_{1} t_{1}^{1-\gamma} h^{m}\left(t_{1}\right) h^{\prime}\left(t_{1}\right)+\int_{t}^{\infty} d t_{1} t_{1}^{-1-\gamma} h^{m}\left(t_{1}\right)
$$

Integrating by parts in the first integral and using (3.51), we obtain

$$
(m+1) \gamma P_{m}^{+}(t)=t^{1-\gamma} h^{m+1}(t)+(1-\gamma) P_{m}^{+}(t)+(m+1) Q_{m}^{+}(t)
$$


namely

$$
((m+2) \gamma-1) P_{m}^{+}(t)=t^{1-\gamma} h^{m+1}(t)+(m+1) Q_{m}^{+}(t)
$$

Substituting that result into the definition of $R_{m}^{+}(t)$, we obtain

$$
\begin{gathered}
((m+2) \gamma-1) R_{m}^{+}(t)=\int_{t}^{\infty} d t_{1} t_{1}^{-1-\gamma} h^{m+1}\left(t_{1}\right)+(m+1) \int_{t}^{\infty} d t_{1} t_{1}^{-1-\gamma} h^{m}\left(t_{1}\right)\left(t^{-1}-t_{1}^{-1}\right) \\
\leq Q_{m+1}^{+}(t)+(m+1) t^{-1} Q_{m}^{+}(t) \\
\leq\left(h(t)+(m+1) t^{-1}\right) Q_{m}^{+}(t)
\end{gathered}
$$

by the monotony of $h$,

$$
\leq(1+(m+1) \gamma) h Q_{m}^{+}(t)
$$

by (3.24). Collecting (3.54) and (3.56) yields (3.47).

\section{Cauchy problem and preliminary asymptotics for the auxiliary system}

In this section, we collect a number of results from I on the Cauchy problem and on the asymptotic behaviour of solutions for the auxiliary system

$$
\left\{\begin{array}{l}
\partial_{t} w=i\left(2 t^{2}\right)^{-1} \Delta w+\left(2 t^{2}\right)^{-1}(2 \nabla \varphi \cdot \nabla+(\Delta \varphi)) w \\
\partial_{t} \varphi=\left(2 t^{2}\right)^{-1}|\nabla \varphi|^{2}+t^{-\gamma} g_{0}(w, w)
\end{array}\right.
$$

Those results are immediate extensions of results contained in I. The main differences are that (i) the results are stated here in terms of $\varphi$ whereas they are stated in I in terms of $s=\nabla \varphi$, and (ii) here we use systematically the estimating functions of time $h_{0}$ and $h$ introduced in Section 3, thereby covering the whole interval $0<\gamma \leq 1$. The proofs will be sketched briefly or omitted.

We first recall the results on the local Cauchy problem with finite initial time (see Proposition I.4.1).

Proposition 4.1. Let $(k, \ell)$ be an admissible pair. Let $t_{0}>0$. Then for any $\left(w_{0}, \varphi_{0}\right) \in H^{k} \oplus Y^{\ell}$, there exist $T_{ \pm}$with $0 \leq T_{-}<t_{0}<T_{+} \leq \infty$ such that : 
(1) The system (4.1) (4.2) has a unique solution $(w, \varphi) \in \mathcal{C}\left(I, H^{k} \oplus Y^{\ell}\right)$ with $(w, \varphi)\left(t_{0}\right)=$ $\left(w_{0}, \varphi_{0}\right)$, where $I=\left(T_{-}, T_{+}\right)$. If $T_{-}>0$ (resp. $\left.T_{+}<\infty\right)$, then $|w(t)|_{k}+|\varphi(t)|_{\ell} \rightarrow \infty$ when $t$ decreases to $T_{-}$(resp. increases to $\left.T_{+}\right)$.

(2) If $\left(w_{0}, \varphi_{0}\right) \in H^{k^{\prime}} \oplus Y^{\ell^{\prime}}$ for some admissible pair $\left(k^{\prime}, \ell^{\prime}\right)$ with $k^{\prime} \geq k$ and $\ell^{\prime} \geq \ell$, then $(w, \varphi) \in \mathcal{C}\left(I, H^{k^{\prime}} \oplus Y^{\ell^{\prime}}\right)$.

(3) For any compact subinterval $J \subset \subset I$, the $\operatorname{map}\left(w_{0}, \varphi_{0}\right) \rightarrow(w, \varphi)$ is continuous from $H^{k-1} \oplus Y^{\ell-1}$ to $L^{\infty}\left(J, H^{k-1} \oplus Y^{\ell-1}\right)$ uniformly on the bounded sets of $H^{k} \oplus Y^{\ell}$, and is pointwise continuous from $H^{k} \oplus Y^{\ell}$ to $L^{\infty}\left(J, H^{k} \oplus Y^{\ell}\right)$.

We next recall the results on the local Cauchy problem in a neighborhood of infinity in time (see Proposition I.5.1).

Proposition 4.2. Let $(k, \ell)$ be an admissible pair. Let $\left(w_{0}, \widetilde{\varphi}_{0}\right) \in H^{k} \oplus Y^{\ell}$ and define $a=\left|w_{0}\right|_{k}$ and $b=\left|\widetilde{\varphi}_{0}\right|_{\ell}$. Then there exists $T_{0}<\infty$, depending on $a, b$, such that for all $t_{0} \geq T_{0}$, there exists $T \leq t_{0}$, depending on $a, b$ and $t_{0}$, such that the system (4.1) (4.2) with initial data $w\left(t_{0}\right)=w_{0}, \varphi\left(t_{0}\right)=h_{0}\left(t_{0}\right) \widetilde{\varphi}_{0}$ has a unique solution $(w, \varphi)$ in the interval $[T, \infty)$ such that $\left(w, h_{0}^{-1} \varphi\right) \in\left(\mathcal{C} \cap L^{\infty}\right)\left([T, \infty), H^{k} \otimes Y^{\ell}\right)$. One can define $T_{0}$ and $T$ by

$$
\begin{aligned}
& C\left(b+a^{2}\right) h\left(T_{0}\right)=1 \\
& T=h_{0}\left(t_{0}\right) h\left(T_{0}\right)^{-1}
\end{aligned}
$$

and the solution $(w, \varphi)$ is estimated for all $t \geq T$ by

$$
\begin{gathered}
|w(t)|_{k} \leq C a \\
|\varphi(t)|_{\ell} \leq C\left(b+a^{2}\right) h_{0}\left(t \vee t_{0}\right) .
\end{gathered}
$$

Sketch of proof. The proof is almost identical with that of Proposition I.5.1 and follows from a priori estimates of the maximal solution obtained from Proposition 4.1. Define $y=|w|_{k}$ and $z=|\varphi|_{\ell}$. By Lemmas 3.2 and 3.3, $y$ and $z$ satisfy

$$
\left\{\begin{array}{l}
\left|\partial_{t} y\right| \leq C t^{-2} y z \\
\left|\partial_{t} z\right| \leq C t^{-2} z^{2}+C t^{-\gamma} y^{2}
\end{array}\right.
$$


For $t \geq t_{0}$, we take $\bar{t}>t_{0}$, we define $Y \equiv Y(\bar{t})=\left\|y ; L^{\infty}\left(\left[t_{0}, \bar{t}\right]\right)\right\|$ and $Z \equiv Z(\bar{t})=$ $\left\|h_{0}(t)^{-1} z ; L^{\infty}\left(\left[t_{0}, \bar{t}\right]\right)\right\|$, we substitute those definitions into (4.7), we integrate over $t$ with the appropriate initial condition and we obtain

$$
\left\{\begin{array}{l}
Y \leq a+C Y Z h\left(t_{0}\right) \\
Z \leq b+C Y^{2}+C Z^{2} h\left(t_{0}\right)
\end{array}\right.
$$

by (3.19) (3.22).

For $t \leq t_{0}$, we take $\bar{t}<t_{0}$, we define $Y \equiv Y(\bar{t})=\left\|y ; L^{\infty}\left(\left[\bar{t}, t_{0}\right]\right)\right\|$ and $Z \equiv Z(\bar{t})=$ $\left\|z ; L^{\infty}\left(\left[\bar{t}, t_{0}\right]\right)\right\|$, we substitute those definitions into (4.7), we integrate over $t$ with the appropriate initial condition and we obtain

$$
\left\{\begin{array}{l}
Y \leq a+C t^{-1} Y Z \\
Z \leq\left(b+C Y^{2}\right) h_{0}\left(t_{0}\right)+C t^{-1} Z^{2}
\end{array}\right.
$$

The proof then proceeds from (4.8) and (4.9) in the same way as that of Proposition I.5.1.

For subsequent applications, we shall need the following lemma, which is essentially identical with Lemma I.5.1.

Lemma 4.1. Let $a>0, b>0, t_{0}>1$ and let $y, z$ be nonnegative continuous functions satisfying $y\left(t_{0}\right)=y_{0}, z\left(t_{0}\right)=z_{0}$ and

$$
\left\{\begin{array}{l}
\left|\partial_{t} y\right| \leq t^{-2} h_{0}(t) b y+t^{-2} a z \\
\left|\partial_{t} z\right| \leq t^{-2} h_{0}(t) b z+t^{-\gamma} \text { a } y .
\end{array}\right.
$$

Define $\bar{y}, \bar{z}$ by

$$
(y, z)=(\bar{y}, \bar{z}) \exp \left(b\left|h(t)-h\left(t_{0}\right)\right|\right) \quad .
$$

Then for $\gamma\left(t_{0}^{\gamma} \wedge t^{\gamma}\right) \geq 2 a^{2}$, the following estimates hold:

$$
\left\{\begin{array}{l}
\bar{y} \leq 2\left(y_{0}+a z_{0} t_{0}^{-1}\right) \\
\bar{z} \leq z_{0}+2 a\left(y_{0}+a z_{0} t_{0}^{-1}\right) h_{0}(t)
\end{array}\right.
$$

for $t \geq t_{0}$, and

$$
\left\{\begin{array}{l}
\bar{y} \leq y_{0}+2 a\left(z_{0}+a y_{0} h_{0}\left(t_{0}\right)\right) t^{-1} \\
\bar{z} \leq 2\left(z_{0}+a y_{0} h_{0}\left(t_{0}\right)\right)
\end{array}\right.
$$


for $1 \leq t \leq t_{0}$

As an easy consequence of Lemma 4.1, we obtain the following uniqueness result at infinity for the system (4.1) (4.2) (see Proposition I.5.2).

Proposition 4.3. Let $(k, \ell)$ be an admissible pair. Let $\left(w_{i}, \varphi_{i}\right), i=1,2$ be two solutions of the system (4.1) (4.2) such that $\left(w_{i}, h_{0}^{-1} \varphi_{i}\right) \in L^{\infty}\left([T, \infty), H^{k} \oplus Y^{\ell}\right)$ for some $T>0$ and such that $\left|w_{1}(t)-w_{2}(t)\right|_{k-1} h_{0}(t)$ and $\left|\varphi_{1}(t)-\varphi_{2}(t)\right|_{\ell-1}$ tend to zero when $t \rightarrow \infty$. Then $\left(w_{1}, \varphi_{1}\right)=\left(w_{2}, \varphi_{2}\right)$.

We finally recall the existence result for the limit of $w(t)$ as $t \rightarrow \infty$ for the solutions of the system (4.1) (4.2) obtained in Proposition 4.2 (see Proposition I.5.3).

Proposition 4.4. Let $(k, \ell)$ satisfy $k \leq \ell+1$ and $\ell>n / 2$. Let $(w, \varphi)$ satisfy (4.1) and be such that $\left(w, h_{0}^{-1} \varphi\right) \in\left(\mathcal{C} \cap L^{\infty}\right)\left([T, \infty), H^{k} \oplus Y^{\ell}\right)$ for some $T>0$. Let

$$
a=\left\|w ; L^{\infty}\left([T, \infty), H^{k}\right)\right\| \quad, \quad b=\left\|h_{0}^{-1} \varphi ; L^{\infty}\left([T, \infty), Y^{\ell}\right)\right\|
$$

Then there exists $w_{+} \in H^{k}$ such that $w(t)$ tends to $w_{+}$strongly in $H^{k-1}$ and weakly in $H^{k}$ when $t \rightarrow \infty$. Furthermore the following estimates hold

$$
\begin{gathered}
\left|w_{+}\right|_{k} \leq a \\
\left|w\left(t_{0}\right)-w(t)\right|_{k-1} \leq C a b h\left(t_{0} \wedge t\right) \\
\left|w(t)-w_{+}\right|_{k-1} \leq C a b h(t)
\end{gathered}
$$

for $t_{0}, t$ sufficiently large, namely $b h\left(t_{0} \wedge t\right) \leq C$ or bh $(t) \leq C$.

\section{$5 \quad$ Existence and properties of the asymptotic dynamics}

In this section we derive the relevant properties of the solutions $\left(w_{m}, \varphi_{m}\right)$ of the system (2.20) (2.21) with initial conditions (2.22) (2.23) and of the solutions $(V, \chi)$ of the transport equations (2.25) (2.26) with initial conditions (2.27). We use systematically the estimating 
functions of time $N_{m}, Q_{m}$ and $P_{m}$ defined by (3.25) (3.26) and (3.31). We begin with the system (2.20) (2.21), which is solved by successive integrations, as explained in Section 2.

Proposition 5.1. Let $(k, \ell)$ be an admissible pair, let $p \geq 0$ be an integer, let $w_{+} \in H^{k+p}$ and let $a=\left|w_{+}\right|_{k+p}$. Let $\left\{w_{0}=w_{+}, w_{m+1}\right\}$ and $\left\{\varphi_{m}\right\}, 0 \leq m \leq p$, be the solution of the system (2.20) (2.21) with initial conditions (2.22) (2.23). Then

(1) $w_{m+1} \in \mathcal{C}\left([1, \infty), H^{k+p-m-1}\right), \varphi_{m} \in \mathcal{C}\left([1, \infty), Y^{\ell+p-m}\right)$ and the following estimates hold for all $t \geq 1$ :

$$
\begin{gathered}
\left|w_{m+1}(t)\right|_{k+p-m-1} \leq A(a) Q_{m}(t) \\
\left|\varphi_{m}(t)\right|_{\ell+p-m} \leq A(a) N_{m}(t)
\end{gathered}
$$

for some estimating function $A(a)$.

If in addition $(p+2) \gamma>1$ and if we define $\varphi_{p+1}$ by (2.21) with initial condition $\varphi_{p+1}(\infty)=0$, then $\varphi_{p+1} \in \mathcal{C}\left([1, \infty), Y^{\ell-1}\right)$ and the following estimate holds :

$$
\left|\varphi_{p+1}(t)\right|_{\ell-1} \leq A(a) P_{p}(t)
$$

(2) The functions $\left\{\varphi_{m}\right\}$ are gauge invariant in the following sense. If $w_{+}^{\prime}=w_{+} \exp (i \sigma)$ for some real valued function $\sigma$ and if $w_{+}^{\prime}$ gives rise to $\left\{\varphi_{m}^{\prime}\right\}$, then $\varphi_{m}^{\prime}=\varphi_{m}$ for $0 \leq m \leq p+1$.

(3) The map $w_{+} \rightarrow\left\{w_{m+1}, \varphi_{m}\right\}$ is uniformly Lipschitz continuous on the bounded sets from the norm topology of $w_{+}$in $H^{k+p}$ to the norms $\left\|Q_{m}^{-1} w_{m+1} ; L^{\infty}\left([1, \infty), H^{k+p-m-1}\right)\right\|$ and $\left\|N_{m}^{-1} \varphi_{m} ; L^{\infty}\left([1, \infty), Y^{\ell+p-m}\right)\right\|, 0 \leq m \leq p$. A similar continuity holds for $\varphi_{p+1}$.

Proof. Part (1). The proof proceeds by induction on $m$. We assume the results to hold for $\left(w_{j}, \varphi_{j}\right)$ for $j \leq m$ and we prove them for $w_{m+1}$ and $\varphi_{m+1}$. We first consider $w_{m+1}$ which is obtained from (2.20). From Lemma 3.2, especially (3.6) with $(k, \ell)$ replaced by $(k+p-m, \ell+$ $p-m)$ which is again an admissible pair and from the induction assumption, we obtain

$$
\left|\partial_{t} w_{m+1}\right|_{k+p-m-1} \leq A(a) t^{-2}\left\{\sum_{0 \leq j \leq m-1} N_{j}(t) Q_{m-j-1}(t)+N_{m}(t)\right\}
$$

Integrating (5.4) between $t$ and $\infty$, using the initial condition $w_{m+1}(\infty)=0$ and using (3.39) (3.35) shows that $w_{m+1} \in \mathcal{C}\left([1, \infty), H^{k+p-m-1}\right)$ and that $w_{m+1}$ satisfies $(5.1)$. 
We next consider $\varphi_{m+1}$ which is obtained from (2.21). From Lemma 3.2, especially (3.7) (3.9) with again $(k, \ell)$ replaced by $(k+p-m, \ell+p-m)$, from the induction assumption and from the result for $w_{m+1}$, we obtain

$\left|\partial_{t} \varphi_{m+1}\right|_{\ell+p-m-1} \leq A(a)\left\{t^{-2} \sum_{0 \leq j \leq m} N_{j}(t) N_{m-j}(t)+t^{-\gamma}\left(\sum_{0 \leq j \leq m-1} Q_{j}(t) Q_{m-1-j}(t)+Q_{m}(t)\right)\right\}$.

Integrating (5.5) between 1 and $t$, using the initial condition $\varphi_{m+1}(1)=0$, and using (3.38) (3.36) and (3.40) (3.43) (3.44) shows that $\varphi_{m+1} \in \mathcal{C}\left([1, \infty), Y^{\ell+p-m-1}\right)$ and that $\varphi_{m+1}$ satisfies $(5.2)$.

We finally assume that $(p+2) \gamma>1$ and estimate $\partial_{t} \varphi_{p+1}$ by $(5.5)$ with $m=p$. The last result and in particular the estimate (5.3) then follow by integration between $t$ and $\infty$ and use of (3.38) (3.37) and (3.40) (3.41) (3.42) with $m=p$.

Part (2). We define for $0 \leq m \leq p+1$

$$
B_{m}=\sum_{0 \leq j \leq m} \bar{w}_{j} w_{m-j}
$$

so that $B_{0}=\left|w_{+}\right|^{2}$ and $B_{m}$ is bounded in time and tends to zero at infinity for $m \geq 1$, for instance in $H_{1}^{1}$ norm. The equation $(2.21)$ for $\varphi_{m+1}$ can be rewritten as

$$
\partial_{t} \varphi_{m+1}=\left(2 t^{2}\right)^{-1} \sum_{0 \leq j \leq m} \nabla \varphi_{j} \cdot \nabla \varphi_{m-j}+t^{-\gamma} \lambda \omega^{\mu-n} B_{m+1}
$$

We next compute

$$
\begin{aligned}
\partial_{t} B_{m+1} & =\left(2 t^{2}\right)^{-1} \sum_{0 \leq j \leq m} 2 \operatorname{Re} \bar{w}_{j} \sum_{0 \leq i \leq m-j}\left(2 \nabla \varphi_{m-i-j} \cdot \nabla+\left(\Delta \varphi_{m-i-j}\right)\right) w_{i} \\
& =t^{-2} \sum_{0 \leq k \leq m}\left(\nabla \varphi_{m-k} \cdot \nabla+\left(\Delta \varphi_{m-k}\right)\right) B_{k} .
\end{aligned}
$$

Using (5.6) and (5.7), we now show by induction on $m$ that $B_{m}$ and $\varphi_{m}$ are gauge invariant. In fact assume that $B_{j}$ and $\varphi_{j}$ are gauge invariant for $j \leq m$. Then $\partial_{t} B_{m+1}$ is gauge invariant by (5.7) and therefore $B_{m+1}$ is gauge invariant because $B_{m+1}(\infty)=0$. Substituting that result into (5.6) and using the induction assumption, we obtain from (5.6) that $\partial_{t} \varphi_{m+1}$ is gauge invariant, and therefore $\varphi_{m+1}$ is gauge invariant since $\varphi_{m+1}(1)=0$ for $m<p$ and $\varphi_{p+1}(\infty)=0$. 
Part (3). Let $\left\{w_{m}, \varphi_{m}\right\}$ and $\left\{w_{m}^{\prime}, \varphi_{m}^{\prime}\right\}$ be the solutions of the system (2.20) (2.21) associated with $w_{+}$and $w_{+}^{\prime}$. From the fact that the RHS of (2.20) (2.21) are bilinear, it follows as in Part (1) by induction on $m$ that the following estimates hold, with $a=\left|w_{+}\right|_{k+p} \vee\left|w_{+}^{\prime}\right|_{k+p}$ :

$$
\begin{gathered}
\left|w_{m+1}-w_{m+1}^{\prime}\right|_{k+p-m-1} \leq A(a)\left|w_{+}-w_{+}^{\prime}\right|_{k+p} Q_{m}(t) \\
\left|\varphi_{m}-\varphi_{m}^{\prime}\right|_{\ell+p-m} \leq A(a)\left|w_{+}-w_{+}^{\prime}\right|_{k+p} N_{m}(t)
\end{gathered}
$$

for $0 \leq m \leq p$, and if $(p+2) \gamma>1$,

$$
\left|\varphi_{p+1}-\varphi_{p+1}^{\prime}\right|_{\ell-1} \leq A(a)\left|w_{+}-w_{+}^{\prime}\right|_{k+p} P_{p}(t)
$$

The continuity as stated in Part (3) follows from those estimates.

Remark 5.1. There is no upper bound on $p$ in Proposition 5.1. However if $(p+1) \gamma>1$, all the $\left(w_{m}, \varphi_{m}\right)$ with $(m+1) \gamma>1$ have the same asymptotic behaviour in time and behave respectively as $t^{-1}$ and Constant as $t \rightarrow \infty$, because $Q_{m}$ and $N_{m}$ saturate to those behaviours in that case.

We define for future reference (see also (2.16) (2.17))

$$
W_{m}=\sum_{0 \leq j \leq m} w_{j} \quad, \quad \phi_{m}=\sum_{0 \leq j \leq m} \varphi_{j}
$$

where $w_{j}, \varphi_{j}$ are obtained by Proposition 5.1.

We now turn to the study of the transport equation

$$
\partial_{t} V=\left(2 t^{2}\right)^{-1}(2 \nabla \phi \cdot \nabla+(\Delta \phi)) V
$$

which we shall use later with $\phi=\phi_{p-1}$, as explained in Section 2 (see (2.25)).

Proposition 5.2. Let $\ell>n / 2$ and $1 \leq k \leq \ell$. Let $T \geq 1, I=[T, \infty)$, let $\phi \in \mathcal{C}\left(I, Y^{\ell}\right)$ with $h_{0}^{-1} \phi \in L^{\infty}\left(I, Y^{\ell}\right)$ and let $w_{+} \in H^{k}$. Then

(1) The equation (5.12) has a solution $V \in\left(\mathcal{C} \cap L^{\infty}\right)\left(I, H^{k}\right)$ which is estimated by

$$
\left\|V: L^{\infty}\left(I, H^{k}\right)\right\| \leq\left|w_{+}\right|_{k} \exp \left(C b \gamma^{-1}\right)
$$


where

$$
b=\left\|h_{0}^{-1} \phi ; L^{\infty}\left(I, Y^{\ell}\right)\right\|,
$$

and which tends to $w_{+}$at infinity in the sense that

$$
\left|V(t)-w_{+}\right|_{k-1} \leq C b \exp \left(C b \gamma^{-1}\right)\left|w_{+}\right|_{k} h(t)
$$

(2) The solution $V$ is unique in $L^{\infty}\left(I, L^{2}\right)$ under the condition that $\left\|V(t)-w_{+}\right\|_{2}$ tends to zero as $t \rightarrow \infty$.

(3) The map $\left(w_{+}, \phi\right) \rightarrow V$ is uniformly Lipschitz continuous in $w_{+}$for the norm topology of $H^{k}$ and is continuous in $\phi$ for the topology of convergence in $Y^{\ell}$ pointwise in $t$ to the norm topology of $L^{\infty}\left(I, H^{k}\right)$ for $h_{0}^{-1} \phi$ in bounded sets of $L^{\infty}\left(I, Y^{\ell}\right)$.

Proof. Part (1). We first take $t_{0} \in I$. Using a regularization (for instance parabolic), energy estimates as in Lemmas 3.2 and 3.3 (see especially (3.6) and (3.12)), and a limiting procedure, one obtains easily the existence of a solution $V_{t_{0}}$ of the equation (5.12) with initial condition $V_{t_{0}}\left(t_{0}\right)=w_{+}$, and such that

$$
V_{t_{0}} \in \mathcal{C}\left(I, H^{k-1}\right) \cap\left(\mathcal{C}_{w} \cap L^{\infty}\right)\left(I, H^{k}\right)
$$

Using the same energy estimates, one then shows that

$$
\begin{gathered}
\left.\left.\left|\partial_{t}\right| V_{t_{0}}(t)\right|_{k}\left|\leq C b t^{-2} h_{0}(t)\right| V_{t_{0}}(t)\right|_{k} \\
\left|\partial_{t}\right| V_{t_{0}}(t)-w_{+}|k-1| \leq C b t^{-2} h_{0}(t)\left(\left|V_{t_{0}}(t)-w_{+}\right|_{k-1}+\left|w_{+}\right|_{k}\right)
\end{gathered}
$$

and for two solutions $V_{t_{0}}$ and $V_{t_{1}}$ associated with $t_{0}$ and $t_{1}$

$$
\left|\partial_{t}\right| V_{t_{0}}(t)-\left.V_{t_{1}}(t)\right|_{k-1}\left|\leq C b t^{-2} h_{0}(t)\right| V_{t_{0}}(t)-\left.V_{t_{1}}(t)\right|_{k-1}
$$

Integrating (5.16) (5.17) between $t_{0}$ and $t$ and integrating (5.18) between $t_{1}$ and $t$, we obtain respectively

$$
\begin{aligned}
\left|V_{t_{0}}(t)\right|_{k} \leq\left|w_{+}\right|_{k} \exp \left(C b\left|h(t)-h\left(t_{0}\right)\right|\right) \leq\left|w_{+}\right|_{k} \exp \left(C b \gamma^{-1}\right) \\
\left|V_{t_{0}}(t)-w_{+}\right|_{k-1} \quad \leq\left|w_{+}\right|_{k}\left(\exp \left(C b\left|h(t)-h\left(t_{0}\right)\right|\right)-1\right) \\
\leq\left|w_{+}\right|_{k} C b \exp \left(C b \gamma^{-1}\right)\left|h(t)-h\left(t_{0}\right)\right|
\end{aligned}
$$




$$
\begin{aligned}
\left|V_{t_{0}}(t)-V_{t_{1}}(t)\right|_{k-1} & \leq\left|V_{t_{0}}\left(t_{1}\right)-w_{+}\right|_{k-1} \exp \left(C b\left|h(t)-h\left(t_{1}\right)\right|\right) \\
& \leq\left|V_{t_{0}}\left(t_{1}\right)-w_{+}\right|_{k-1} \exp \left(C b \gamma^{-1}\right) .
\end{aligned}
$$

Substituting (5.20) into (5.21) yields

$$
\left|V_{t_{0}}(t)-V_{t_{1}}(t)\right|_{k-1} \leq\left|w_{+}\right|_{k} C b \exp \left(2 C b \gamma^{-1}\right)\left|h\left(t_{1}\right)-h\left(t_{0}\right)\right|
$$

It follows from (5.22) that when $t_{0} \rightarrow \infty, V_{t_{0}}$ has a limit $V \in\left(\mathcal{C} \cap L^{\infty}\right)\left(I, H^{k-1}\right)$ satisfying (5.15). One sees easily that $V$ satisfies the equation (5.12). From the estimate (5.19) it follows by a standard compactness argument that $V \in\left(\mathcal{C}_{w} \cap L^{\infty}\right)\left(I, H^{k}\right)$ and that $V$ satisfies the estimate (5.13). Furthermore $V$ also satisfies (5.16) so that $|V(t)|_{k}$ is Lipschitz continuous in $t$, which together with weak continuity in $H^{k}$ implies strong continuity in $H^{k}$.

Part (2). If $V_{1}$ and $V_{2}$ are two solutions of (5.12) one obtains by the same energy estimates as above

$$
\left\|V_{1}(t)-V_{2}(t)\right\|_{2} \leq\left\|V_{1}\left(t^{\prime}\right)-V_{2}\left(t^{\prime}\right)\right\|_{2} \exp \left(C b\left|h(t)-h\left(t^{\prime}\right)\right|\right) \quad .
$$

Taking the limit $t^{\prime} \rightarrow \infty$ shows that $V_{1}=V_{2}$.

Part (3). Continuity of $V$ with respect to $w_{+}$follows immediately from the linearity of the equation (5.12) and from the estimate (5.13). In order to prove continuity with respect to $\phi$, we first derive an estimate for the difference of two solutions $V_{1}$ and $V_{2}$ associated with $\phi_{1}$ and $\phi_{2}$. We assume that $\phi_{1} \in \mathcal{C}\left(I, Y^{\ell+1}\right)$ with $h_{0}^{-1} \phi \in L^{\infty}\left(I, Y^{\ell+1}\right)$, that $\phi_{2} \in \mathcal{C}\left(I, Y^{\ell}\right)$ with $h_{0}^{-1} \phi_{2} \in L^{\infty}\left(I, Y^{\ell}\right)$, that $V_{1} \in\left(\mathcal{C} \cap L^{\infty}\right)\left(I, H^{k+1}\right)$ and that $V_{2} \in\left(\mathcal{C} \cap L^{\infty}\right)\left(I, H^{k}\right)$. Let $V_{-}=V_{1}-V_{2}$ and $\phi_{-}=\phi_{1}-\phi_{2}$. It follows from (5.12) that

$$
\partial_{t} V_{-}=\left(2 t^{2}\right)^{-1}\left\{\left(2 \nabla \phi_{2} \cdot \nabla+\left(\Delta \phi_{2}\right)\right) V_{-}+\left(2 \nabla \phi_{-} \cdot \nabla+\left(\Delta \phi_{-}\right)\right) V_{1}\right\} \quad .
$$

Let

$$
a=\operatorname{Max}_{i=1,2}\left\|V_{i} ; L^{\infty}\left(I, H^{k}\right)\right\| \quad, \quad b=\operatorname{Max}_{i=1,2}\left\|h_{0}^{-1} \phi_{i} ; L^{\infty}\left(I, Y^{\ell}\right)\right\| \quad .
$$

Estimating (5.24) by Lemma 3.5, we obtain

$$
\begin{aligned}
\left|\partial_{t}\right| V_{-}|k| & \leq C t^{-2}\left\{\left|\phi_{2}\right|_{\ell}\left|V_{-}\right|_{k}+\left|\phi_{-}\right|_{\ell}\left|V_{1}\right|_{k}+\left|\phi_{-}\right|_{*}\left|V_{1}\right|_{k+1}\right\} \\
& \leq C t^{-2}\left\{b h_{0}\left|V_{-}\right|_{k}+a\left|\phi_{-}\right|_{\ell}+\left|\phi_{-}\right|_{*}\left|V_{1}\right|_{k+1}\right\}
\end{aligned}
$$


where $|f|_{*} \equiv\|\nabla f\|_{\infty}$. On the other hand, by Lemma 3.4 we obtain

$$
\begin{aligned}
\left.\left|\partial_{t}\right| V_{1}\right|_{k+1} \mid & \leq C t^{-2}\left\{\left|\phi_{1}\right|_{\ell}\left|V_{1}\right|_{k+1}+\left|\phi_{1}\right|_{\ell+1}\left|V_{1}\right|_{k}\right\} \\
& \leq C t^{-2}\left\{b h_{0}\left|V_{1}\right|_{k+1}+a\left|\phi_{1}\right|_{\ell+1}\right\}
\end{aligned}
$$

Integrating (5.26) between $t_{0}$ and $t$ and using the fact that

$$
\left|\partial_{t} y\right| \leq C_{0} t^{-2} h_{0} y+z
$$

implies

$$
\begin{aligned}
y(t) & \leq y\left(t_{0}\right) \exp \left(C_{0}\left|h(t)-h\left(t_{0}\right)\right|\right)+\left|\int_{t_{0}}^{t} d t_{1} z\left(t_{1}\right) \exp \left(C_{0}\left|h(t)-h\left(t_{1}\right)\right|\right)\right| \\
& \leq \exp \left(C_{0} \gamma^{-1}\right)\left(y\left(t_{0}\right)+\left|\int_{t_{0}}^{t} d t_{1} z\left(t_{1}\right)\right|\right),
\end{aligned}
$$

we obtain

$$
\left|V_{1}(t)\right|_{k+1} \leq C\left(\left|V_{1}\left(t_{0}\right)\right|_{k+1}+\left.\left|\int_{t_{0}}^{t} d t_{1} t_{1}^{-2}\right| \phi_{1}\left(t_{1}\right)\right|_{\ell+1} \mid\right)
$$

where $C$ depends on $a, b$. Substituting (5.29) into (5.25) and integrating between $t_{0}$ and $t$ yields similarly

$$
\begin{aligned}
\left|V_{-}(t)\right|_{k} \leq C\{ & \left|V_{-}\left(t_{0}\right)\right|_{k}+\left.\left.\left.\left|\int_{t_{0}}^{t} d t_{1} t_{1}^{-2}\right| \phi_{-}\left(t_{1}\right)\right|_{\ell}|+| V_{1}\left(t_{0}\right)\right|_{k+1}\left|\int_{t_{0}}^{t} d t_{1} t_{1}^{-2}\right| \phi_{-}\left(t_{1}\right)\right|_{*} \mid \\
& \left.+\left.\left|\int_{t_{0}}^{t} d t_{1} t_{1}^{-2}\right| \phi_{-}\left(t_{1}\right)\right|_{*} \int_{t_{0}}^{t_{1}} d t_{2} t_{2}^{-2}\left|\phi_{1}\left(t_{2}\right)\right|_{\ell+1} \mid\right\} .
\end{aligned}
$$

In particular if $V_{1}$ and $V_{2}$ are solutions of (5.12) with $\phi_{1}$ and $\phi_{2}$ respectively and with initial data $w_{+1} \in H^{k+1}$ and $w_{+2} \in H^{k}$ at time $t_{0}$, as obtained in Part (1), then the following estimate holds uniformly in $t_{0}$ and $t$

$$
\begin{gathered}
\left|V_{-}(t)\right|_{k} \leq C\left\{\left|w_{+1}-w_{+2}\right|_{k}+\int_{1}^{\infty} d t t^{-2}\left|\phi_{-}(t)\right|_{\ell}\right. \\
\left.+\left(\left|w_{+1}\right|_{k+1}+\int_{1}^{\infty} d t t^{-2}\left|\phi_{1}(t)\right|_{\ell+1}\right) \int_{1}^{\infty} d t t^{-2}\left|\phi_{-}(t)\right|_{*}\right\},
\end{gathered}
$$

where all the integrals are convergent under the assumptions made on $\phi_{1}$ and $\phi_{2}$.

We can now prove the continuity with respect to $\phi$. The proof proceeds as in Step 7 of that of Proposition I.4.1. We introduce a regularization defined as follows. We choose a function $\psi_{1} \in \mathcal{S}\left(\mathbb{R}^{n}\right)$ such that $\int d x \psi_{1}(x)=1$ and such that $\left.|\xi|^{-2}\left(\widehat{\psi}_{1}(\xi)-1\right)\right|_{\xi=0}=0$. We define 
$\psi_{\varepsilon}(x)=\varepsilon^{-n} \psi_{1}(x / \varepsilon)$, so that $\widehat{\psi}_{\varepsilon}(\xi)=\widehat{\psi}_{1}(\varepsilon \xi)$ and we define the regularization by $f \rightarrow f_{\varepsilon}=\psi_{\varepsilon} * f$ for all $f \in \mathcal{S}^{\prime}$. An immediate computation yields

$$
\left\|\partial f_{\varepsilon}\right\|_{2} \leq\left\|\partial \psi_{\varepsilon}\right\|_{1}\|f\|_{2}=\varepsilon^{-1}\left\|\partial \psi_{1}\right\|_{1}\|f\|_{2}
$$

and

$$
\left\|f_{\varepsilon}-f\right\|_{\infty} \leq\left\|\left(\widehat{\psi}_{\varepsilon}-1\right) \hat{f}\right\|_{1} \leq \varepsilon^{\theta}\left\||\xi|^{-n / 2-\theta}\left(\widehat{\psi}_{1}(\xi)-1\right)\right\|_{2}\left\|f ; \dot{H}^{n / 2+\theta}\right\| \quad .
$$

Let now $w_{+} \in H^{k}$ and $\phi, \phi^{\prime} \in \mathcal{C}\left(I, Y^{\ell}\right)$ with $h_{0}^{-1} \phi, h_{0}^{-1} \phi^{\prime} \in L^{\infty}\left(I, Y^{\ell}\right)$ and such that

$$
\left\|h_{0}^{-1} \phi ; L^{\infty}\left(I, Y^{\ell}\right)\right\| \vee\left\|h_{0}^{-1} \phi^{\prime} ; L^{\infty}\left(I, Y^{\ell}\right)\right\| \leq b
$$

Let $V$ and $V^{\prime}$ be the solutions of the equation (5.12) with $\phi$ and $\phi^{\prime}$ respectively and with initial data $w_{+}$at $t_{0}$ obtained in Part (1). We regularize $w_{+}, \phi, \phi^{\prime}$ to $w_{+\varepsilon}, \phi_{\varepsilon}, \phi_{\varepsilon}^{\prime}$, so that the following estimates hold :

$$
\left|w_{+\varepsilon}\right|_{k+1} \leq C \varepsilon^{-1}\left|w_{+}\right|_{k} \quad, \quad\left|\phi_{\varepsilon}\right|_{\ell+1} \leq C \varepsilon^{-1}|\phi|_{\ell} \quad, \quad\left|\phi_{\varepsilon}^{\prime}\right|_{\ell+1} \leq C \varepsilon^{-1}\left|\phi^{\prime}\right|_{\ell}
$$

and

$$
\left|\phi-\phi_{\varepsilon}\right|_{*} \leq C \varepsilon^{3 / 2}|\phi|_{\ell} \quad, \quad\left|\phi^{\prime}-\phi_{\varepsilon}^{\prime}\right|_{*} \leq C \varepsilon^{3 / 2}\left|\phi^{\prime}\right|_{\ell} .
$$

The estimates (5.34) follow from (5.32) and the estimates (5.35) follow from (5.33) and from the definition of $Y^{\ell}$. Let $V_{\varepsilon}$ and $V_{\varepsilon}^{\prime}$ be the solutions of (5.12) obtained from $\left(w_{+\varepsilon}, \phi_{\varepsilon}\right)$ and $\left(w_{+\varepsilon}^{\prime}, \phi_{\varepsilon}^{\prime}\right)$. We estimate

$$
\left|V(t)-V^{\prime}(t)\right|_{k} \leq\left|V(t)-V_{\varepsilon}(t)\right|_{k}+\left|V_{\varepsilon}(t)-V_{\varepsilon}^{\prime}(t)\right|_{k}+\left|V_{\varepsilon}^{\prime}(t)-V^{\prime}(t)\right|_{k} \quad .
$$

We estimate the three norms in the RHS of (5.36) by applying successively $(5.31)$ with $\left(V_{1}, V_{2}\right)=$ $\left(V_{\varepsilon}, V\right),\left(V_{\varepsilon}, V_{\varepsilon}^{\prime}\right)$ and $\left(V_{\varepsilon}^{\prime}, V^{\prime}\right)$. We obtain

$$
\begin{gathered}
\left|V(t)-V^{\prime}(t)\right|_{k} \leq C\left\{\left|w_{+}-w_{+\varepsilon}\right|_{k}+\int_{1}^{\infty} d t t^{-2}\left(\left|\phi(t)-\phi_{\varepsilon}(t)\right|_{\ell}+\left|\phi_{\varepsilon}(t)-\phi_{\varepsilon}^{\prime}(t)\right|_{\ell}\right.\right. \\
\left.\left.+\left|\phi_{\varepsilon}^{\prime}(t)-\phi^{\prime}(t)\right|_{\ell}\right)+\varepsilon^{-1} \int_{1}^{\infty} d t t^{-2}\left(\left|\phi(t)-\phi_{\varepsilon}(t)\right|_{*}+\left|\phi_{\varepsilon}(t)-\phi_{\varepsilon}^{\prime}(t)\right|_{*}+\left|\phi_{\varepsilon}^{\prime}(t)-\phi^{\prime}(t)\right|_{*}\right)\right\}
\end{gathered}
$$

where we have used (5.34). Using the inequalities

$$
\begin{aligned}
& \left|\phi_{\varepsilon}-\phi_{\varepsilon}^{\prime}\right|_{\ell} \leq\left|\phi-\phi^{\prime}\right|_{\ell} \\
& \left|\phi_{\varepsilon}-\phi_{\varepsilon}^{\prime}\right|_{*} \leq\left|\phi-\phi^{\prime}\right|_{*} \leq\left|\phi-\phi^{\prime}\right|_{\ell} \\
& \left|\phi_{\varepsilon}^{\prime}-\phi^{\prime}\right|_{\ell} \leq\left|\phi_{\varepsilon}-\phi\right|_{\ell}+2\left|\phi-\phi^{\prime}\right|_{\ell}
\end{aligned}
$$


and (5.35), we can continue (5.37) by

$$
\begin{aligned}
\left|V(t)-V^{\prime}(t)\right|_{k} & \leq C\left\{\left|w_{+}-w_{+\varepsilon}\right|_{k}+\int_{1}^{\infty} d t t^{-2}\left|\phi(t)-\phi_{\varepsilon}(t)\right|_{\ell}+\varepsilon^{1 / 2}\right. \\
& \left.+\left(1+\varepsilon^{-1}\right) \int_{1}^{\infty} d t t^{-2}\left|\phi(t)-\phi^{\prime}(t)\right|_{\ell}\right\} .
\end{aligned}
$$

For fixed $\phi$, by the Lebesgue dominated convergence theorem, the first integral in the RHS tends to zero when $\varepsilon \rightarrow 0$, while the second integral tends to zero when $\phi^{\prime} \rightarrow \phi$ in $Y^{\ell}$ pointwise in $t$. The RHS of (5.38) can then be made arbitrarily small by first taking $\varepsilon$ sufficiently small and then letting $\phi^{\prime}$ tend to $\phi$ in the previous sense for fixed $\varepsilon$.

We next turn to the analogous transport equation

$$
\partial_{t} \chi=t^{-2} \nabla \phi \cdot \nabla \chi
$$

which we shall use together with (5.12), as explained in Section 2.

Proposition 5.3. Let $\ell>n / 2$. Let $T \geq 1, I=[T, \infty)$, let $\phi \in \mathcal{C}\left(I, Y^{\ell+1}\right)$ with $h_{0}^{-1} \phi \in$ $L^{\infty}\left(I, Y^{\ell+1}\right)$ and let $\psi_{+} \in Y^{\ell}$. Then

(1) The equation (5.39) has a solution $\chi \in\left(\mathcal{C} \cap L^{\infty}\right)\left(I, Y^{\ell}\right)$ which is estimated by

$$
\left\|\chi ; L^{\infty}\left(I, Y^{\ell}\right)\right\| \leq\left|\psi_{+}\right|_{\ell} \exp \left(C b \gamma^{-1}\right)
$$

where

$$
b=\left\|h_{0}^{-1} \phi ; L^{\infty}\left(I, Y^{\ell+1}\right)\right\|,
$$

and tends to $\psi_{+}$at infinity in the sense that

$$
\left|\chi(t)-\psi_{+}\right|_{\ell-1} \leq C b \exp \left(C b \gamma^{-1}\right)\left|\psi_{+}\right|_{\ell} h(t)
$$

(2) The solution $\chi$ is unique in $L^{\infty}\left(I, L^{\infty}\right)$ under the condition that $\left\|\chi(t)-\psi_{+}\right\|_{\infty}$ tends to zero as $t \rightarrow \infty$.

(3) The map $\left(\psi_{+}, \phi\right) \rightarrow \chi$ is uniformly Lipschitz continuous in $\psi_{+}$for the norm topology of $Y^{\ell}$ and is continuous in $\phi$ for the topology of convergence in $Y^{\ell+1}$ pointwise in $t$ to the norm topology of $L^{\infty}\left(I, Y^{\ell}\right)$, for $h_{0}^{-1} \phi$ in bounded sets of $L^{\infty}\left(I, Y^{\ell+1}\right)$. 
(4) Let in addition $w_{+} \in H^{k}$ and let $V$ be the solution of (5.12) obtained in Proposition 5.2. Then for fixed $\phi, V \exp (-i \chi)$ is gauge invariant in the following sense : if $(V, \chi)$ and $\left(V^{\prime}, \chi^{\prime}\right)$ are the solutions obtained from $\left(w_{+}, \psi_{+}\right)$and $\left(w_{+}^{\prime}, \psi_{+}^{\prime}\right)$ and if $w_{+} \exp \left(-i \psi_{+}\right)=w_{+}^{\prime} \exp \left(-i \psi_{+}^{\prime}\right)$, then $V(t) \exp (-i \chi(t))=V^{\prime}(t) \exp \left(-i \chi^{\prime}(t)\right)$ for all $t \in I$.

Proof. Parts (1) (2) (3). The proof is the same as that of Proposition 5.2, starting from the estimates (3.13) (3.14) (3.16) (3.18) of Lemmas 3.3, 3.4 and 3.5.

Part (4). It follows from (5.12) and (5.39) that $V \exp (-i \chi)$ also satisfies (5.12), with gauge invariant initial condition $V(\infty) \exp (-i \chi(\infty))=w_{+} \exp \left(-i \psi_{+}\right)$. The result then follows from the uniqueness statement of Proposition 5.2, part (2).

In the subsequent applications, we shall use the solutions of the equations (5.12) and (5.39) associated with $\phi=\phi_{p-1}$ defined by (5.11) (see (2.25) (2.26)). In particular we shall use $V$ as a substitute for $W_{p}$, also defined by (5.11), and we shall need the fact that $V$ is a sufficiently good approximation of $W_{p}$. We collect the relevant properties in the following proposition.

Proposition 5.4. Let $(k, \ell)$ be an admissible pair. Let $p \geq 1$ be an integer. Let $w_{+} \in H^{k+p+1}$, let $a=\left|w_{+}\right|_{k+p+1}$ and let $\phi=\phi_{p-1}$ be defined by (5.11) and Proposition 5.1, so that $h_{0}^{-1} \phi \in$ $\left(\mathcal{C} \cap L^{\infty}\right)\left([1, \infty), Y^{\ell+2}\right)$. Let $V$ be the solution of (5.12) defined by Proposition 5.2, so that $V \in\left(\mathcal{C} \cap L^{\infty}\right)\left([1, \infty), H^{k+2}\right)$.

(1) Let $W_{p}$ be defined by (5.11) and Proposition 5.1 so that $W_{p} \in\left(\mathcal{C} \cap L^{\infty}\right)\left([1, \infty), H^{k+1}\right)$. Then

$$
\left|V(t)-W_{p}(t)\right|_{k} \leq A(a) Q_{p}(t)
$$

for some estimating function $A(a)$.

(2) Let $\psi_{+} \in Y^{\ell+1}$ and let $\chi$ be the solution of (5.39) defined by Proposition 5.3, so that $\chi \in\left(\mathcal{C} \cap L^{\infty}\right)\left([1, \infty), Y^{\ell+1}\right)$. Then

$$
\left|\chi(t)-\psi_{+}\right|_{\ell} \leq A(a)\left|\psi_{+}\right|_{\ell+1} h(t)
$$

and $V(t) \exp (-i \chi(t))$ is gauge invariant. 
(3) The map $w_{+} \rightarrow V$ is continuous from $H^{k+p+1}$ to $L^{\infty}\left([1, \infty), H^{k+2}\right)$ and the map $\left(w_{+}, \psi_{+}\right) \rightarrow$ $\chi$ is continuous from $H^{k+p+1} \oplus Y^{\ell+1}$ to $L^{\infty}\left([1, \infty), Y^{\ell+1}\right)$.

Proof. Part (1). From (2.20) and (5.11) it follows that

$$
\partial_{t} W_{p}=\left(2 t^{2}\right)^{-1}\left\{\left(2 \nabla \phi_{p-1} \cdot \nabla+\left(\Delta \phi_{p-1}\right)\right) W_{p}-\sum_{\substack{i \leq p-1, j \leq p \\ i+j \geq p}}\left(2 \nabla \varphi_{i} \cdot \nabla+\left(\Delta \varphi_{i}\right)\right) w_{j}\right\}
$$

so that

$$
\partial_{t}\left(V-W_{p}\right)=\left(2 t^{2}\right)^{-1}\left\{\left(2 \nabla \phi_{p-1} \cdot \nabla+\left(\Delta \phi_{p-1}\right)\right)\left(V-W_{p}\right)+\sum_{\substack{i \leq p-1, j \leq p \\ i+j \geq p}}\left(2 \nabla \varphi_{i} \cdot \nabla+\left(\Delta \varphi_{i}\right)\right) w_{j}\right\} .
$$

From Lemma 3.3, esp. (3.12) and Lemma 3.2, esp. (3.6), we obtain

$$
\left|\partial_{t}\right| V-\left.W_{p}\right|_{k} \mid \leq C\left\{t^{-2} h_{0}(t) b\left|V-W_{p}\right|_{k}+t^{-2} \sum_{\substack{i \leq p-1, j \leq p \\ i+j \geq p}}\left|\varphi_{i}\right|_{\ell}\left|w_{j}\right|_{k+1}\right\}
$$

where

$$
b=\left\|h_{0}^{-1} \phi_{p-1} ; L^{\infty}\left([1, \infty), Y^{\ell}\right)\right\| \quad .
$$

Integrating (5.46) between $t$ and infinity, using (5.1) (5.2) (5.27) (5.28), we obtain

$$
\begin{aligned}
\left|V(t)-W_{p}(t)\right|_{k} & \leq A(a) \int_{t}^{\infty} d t_{1} t_{1}^{-2} \sum_{\substack{i \leq p-1, j \leq p \\
i+j \geq p}} N_{i}\left(t_{1}\right) Q_{j-1}\left(t_{1}\right) \\
& \leq A(a) \sum_{p \leq m \leq 2 p-1} Q_{m}(t) \leq A(a) Q_{p}(t)
\end{aligned}
$$

by (3.39) (3.35) and (3.28).

Part (2) is a partial rewriting of Proposition 5.3 in the special case $\phi=\phi_{p-1}$.

Part (3). The continuity properties stated there follow by combining those of Propositions 5.1, part (3), 5.2 part (3) and 5.3, part (3).

Remark 5.2. By keeping track of the orders of derivation more accurately, one sees easily that Proposition 5.4 holds with $(k, \ell)$ replaced everywhere by $(k-1, \ell-1)$. We have stated Proposition 5.4 at the level of regularity which will be used in the subsequent applications. 


\section{Asymptotics and wave operators for the auxiliary sys- tem}

In this section we derive the main technical results of this paper. We prove that sufficiently regular solutions $(w, \varphi)$ of the auxiliary system (4.1) (4.2) have asymptotic states $\left(w_{+}, \psi_{+}\right)$, and conversely that sufficiently regular asymptotic states $\left(w_{+}, \psi_{+}\right)$generate solutions $(w, \varphi)$ of the auxiliary system in the sense described in Section 2, thereby allowing for the definition of the wave operator $\Omega_{0}:\left(w_{+}, \psi_{+}\right) \rightarrow(w, \varphi)$.

We first prove the existence of asymptotic states of solutions $(w, \varphi)$ of $(4.1)(4.2)$. The existence of $w_{+}$is already established in Proposition 4.4 under rather general assumptions. However the existence of $\psi_{+}$requires a more complicated construction and stronger assumptions.

Proposition 6.1. Let $(k, \ell)$ be an admissible pair. Let $p \geq 0$ be an integer. Let $T \geq 2$, $I=[T, \infty)$, let $(w, \varphi)$ be a solution of the system (4.1) (4.2) such that $\left(w, h_{0}^{-1} \varphi\right) \in(\mathcal{C} \cap$ $\left.L^{\infty}\right)\left(I, H^{k+(p+1) \vee 2} \oplus Y^{\ell+p}\right)$ and let

$$
a=\left\|w ; L^{\infty}\left([T, \infty), H^{k+(p+1) \vee 2}\right)\right\| \quad, \quad b=\left\|h_{0}^{-1} \varphi ; L^{\infty}\left([T, \infty), Y^{\ell+p}\right)\right\| \quad .
$$

Let $w_{+}=\lim _{t \rightarrow \infty} w(t) \in H^{k+p+1}$ be defined by Proposition 4.4. Let $\left\{w_{m+1}, \varphi_{m}\right\}, 0 \leq m \leq p$ be defined by Proposition 5.1, and let $W_{m}, \phi_{m}, 0 \leq m \leq p$, be defined by (5.11). Then the following estimates hold for all $t \in I$ :

$$
\begin{gathered}
\left|w(t)-W_{m}(t)\right|_{k+p-m-1} \leq A(a, b) Q_{m}(t) \\
\left|\varphi(t)-\phi_{m}(t)\right|_{\ell+p-m-1} \leq A(a, b) N_{m+1}(t)
\end{gathered}
$$

for $0 \leq m \leq p$, and for some estimating function $A(a, b)$.

If in addition $(p+2) \gamma>1$, then the following limit exists

$$
\lim _{t \rightarrow \infty}\left(\varphi(t)-\phi_{p}(t)\right)=\psi_{+}
$$

as a strong limit in $Y^{\ell-1}$, and the following estimate holds

$$
\left|\varphi(t)-\phi_{p}(t)-\psi_{+}\right|_{\ell-1} \leq A(a, b) P_{p}(t)
$$


Proof. The proof proceeds by induction on $m$. For $0 \leq m \leq p$, we define

$$
\begin{aligned}
& q_{m+1}(t)=w(t)-W_{m}(t) \\
& \psi_{m+1}(t)=\varphi(t)-\phi_{m}(t)
\end{aligned}
$$

We also define $q_{0}=w$ and $\psi_{0}=\varphi$. We assume that the estimates (6.2) (6.3) hold for $\left(q_{j}, \psi_{j}\right)$, $0 \leq j \leq m$ and we derive them for $\left(q_{m+1}, \psi_{m+1}\right)$.

We substitute the decompositions $w=W_{m}+q_{m+1}$ and $\varphi=\phi_{m}+\psi_{m+1}$ in the LHS of (4.1) (4.2) and we partly substitute the decompositions $w=W_{m-1}+q_{m}$ and $\varphi=\phi_{m-1}+\psi_{m}$ in the RHS of the same equations. Using in addition (2.20) (2.21), we obtain

$$
\begin{gathered}
\partial_{t} q_{m+1}=\left(2 t^{2}\right)^{-1}\left\{i \Delta w+(2 \nabla \varphi \cdot \nabla+(\Delta \varphi)) q_{m}\right. \\
\left.+\left(2 \nabla \psi_{m} \cdot \nabla+\left(\Delta \psi_{m}\right)\right) W_{m-1}+\sum_{\substack{0 \leq i, j \leq m-1 \\
i+j \geq m}}\left(2 \nabla \varphi_{i} \cdot \nabla+\left(\Delta \varphi_{i}\right)\right) w_{j}\right\} \\
\partial_{t} \psi_{m+1}=\left(2 t^{2}\right)^{-1}\left\{\left(\nabla \varphi+\nabla \phi_{m-1}\right) \cdot \nabla \psi_{m}+\sum_{\substack{0 \leq i, j \leq m-1 \\
i+j \geq m}} \nabla \varphi_{i} \cdot \nabla \varphi_{j}\right\} \\
+t^{-\gamma}\left\{g_{0}\left(q_{m}, q_{1}\right)+g_{0}\left(q_{m}, W_{m-1}-w_{0}\right)+2 g_{0}\left(q_{m+1}, w_{0}\right)+\sum_{\substack{0 \leq i, j \leq m-1 \\
i+j \geq m+1}} g_{0}\left(w_{i}, w_{j}\right)\right\} .
\end{gathered}
$$

The equation (6.9) holds only for $m \geq 1$ and the last bracket thereof has been obtained by using the fact that

$$
g_{0}\left(q_{m}, w+W_{m-1}\right)-2 g_{0}\left(w_{m}, w_{0}\right)=g_{0}\left(q_{m}, q_{1}\right)+g_{0}\left(q_{m}, W_{m-1}-w_{0}\right)+2 g_{0}\left(q_{m+1}, w_{0}\right)
$$

For $m=0,(6.9)$ should be replaced by

$$
\partial_{t} \psi_{1}=\left(2 t^{2}\right)^{-1}|\nabla \varphi|^{2}+t^{-\gamma}\left(g_{0}\left(q_{1}, q_{1}\right)+2 g_{0}\left(q_{1}, w_{0}\right)\right)
$$

We estimate the RHS of (6.8) (6.9) by Lemma 3.2 with $(k, \ell)$ replaced by $(k+p-m, \ell+p-m)$, which is again an admissible pair. We use Proposition 5.1 to estimate $W_{m-1}, w_{j}, \phi_{m-1}$ and $\varphi_{j}$, and we use the induction hypothesis to estimate $q_{m}, q_{1}, q_{m+1}$ and $\psi_{m}$. Note that $W_{m-1}-w_{0}$, which occurs only for $m \geq 2$, satisfies the same estimate as $q_{1}$. In the induction procedure, as in the proof of Proposition 5.1, one has first to complete the estimation of $q_{m+1}$ before estimating $\psi_{m+1}$. One then obtains

$$
\left|\partial_{t} q_{m+1}\right|_{k+p-m-1} \leq A(a, b) t^{-2}\left\{1+h_{0} Q_{m-1}+N_{m}+\sum_{m \leq i+j \leq 2(m-1)} N_{i} Q_{j-1}\right\}
$$




$$
\begin{gathered}
\left|\partial_{t} \psi_{m+1}\right|_{\ell+p-m-1} \leq A(a, b)\left\{t^{-2}\left(h_{0} N_{m}+\sum_{m \leq i+j \leq 2(m-1)} N_{i} N_{j}\right)\right. \\
\left.+t^{-\gamma}\left(h Q_{m-1}+Q_{m}+\sum_{m+1 \leq i+j \leq 2(m-1)} Q_{i-1} Q_{j-1}\right)\right\}
\end{gathered}
$$

for $m \geq 1$, and

$$
\begin{aligned}
& \left|\partial_{t} q_{1}\right|_{k+p-1} \leq A(a, b) t^{-2}\left(1+h_{0}\right) \\
& \left|\partial_{t} \psi_{1}\right|_{\ell+p-1} \leq A(a, b)\left\{t^{-2} h_{0}^{2}+t^{-\gamma} h\right\} .
\end{aligned}
$$

Integrating (6.10) between $t$ and infinity with the condition $q_{m+1}(\infty)=0$ which follows from the definition and using (3.39) (3.35) (3.28) and the fact that $t^{-1} Q_{m}(1) \leq Q_{m}(t)$ yields (6.2).

Integrating (6.11) between $T$ and $t$, using (3.38) (3.36) (3.27) for the first bracket and (3.40) (3.43) (3.44) (3.28) for the second bracket yields

$$
\begin{aligned}
\left|\psi_{m+1}(t)\right|_{\ell+p-m-1} & \leq C+A(a, b) N_{m+1}(t) \\
& \leq\left(C N_{m+1}(T)^{-1}+A(a, b)\right) N_{m+1}(t)
\end{aligned}
$$

with

$$
C=\left|\psi_{m+1}(T)\right|_{\ell+p-m-1}
$$

where we have used the fact that $N_{m+1}$ is increasing in $T$, and we have assumed that $T$ is bounded away from 1. This yields (6.3).

We next turn to the proof of (6.5). In that case, the RHS of (6.11) with $m=p$ is integrable in time, which proves the existence of the limit (6.4). Integrating (6.11) between $t$ and infinity and using (3.38) (3.37) for the first bracket and (3.40) (3.41) (3.42) (3.28) for the second bracket yields (6.5).

We now turn to the construction of solutions $(w, \varphi)$ of the system (4.1) (4.2) with given asymptotic states $\left(w_{+}, \psi_{+}\right)$. For that purpose we first take a (large) positive $t_{0}$ and we construct the solution $\left(w_{t_{0}}, \varphi_{t_{0}}\right)$ of (4.1) (4.2) with initial data $\left(V\left(t_{0}\right), \phi_{p}\left(t_{0}\right)+\chi\left(t_{0}\right)\right)$ at $t_{0}$. The solution $(w, \varphi)$ will then be obtained therefrom by taking the limit $t_{0} \rightarrow \infty$, as explained in Section 2 .

Proposition 6.2. Let $(k, \ell)$ be an admissible pair and let $p$ be an integer such that $(p+2) \gamma>1$. Let $w_{+} \in H^{k+(p+1) \vee 2}$ and $\psi_{+} \in Y^{\ell+1}$. Let $\phi=\phi_{p-1}$ be defined by (5.11) and Proposition 5.1, 
so that $h_{0}^{-1} \phi \in\left(\mathcal{C} \cap L^{\infty}\right)\left([1, \infty), Y^{\ell+2}\right)$. Let $V$ and $\chi$ be the solutions of (5.12) and (5.39) respectively, obtained in Propositions 5.4, so that $(V, \chi) \in\left(\mathcal{C} \cap L^{\infty}\right)\left([1, \infty), H^{k+2} \oplus Y^{\ell+1}\right)$. Let

$$
a_{+}=\left|w_{+}\right|_{k+(p+1) \vee 2} \quad, \quad b_{+}=\left|\psi_{+}\right|_{\ell+1} \quad .
$$

Then there exist $T_{0}$ and $T, 1 \leq T_{0}, T<\infty$, depending only on $\left(\gamma, p, a_{+}, b_{+}\right)$such that for all $t_{0} \geq T_{0} \vee T$, the system (4.1) (4.2) with initial data $w_{t_{0}}\left(t_{0}\right)=V\left(t_{0}\right), \varphi_{t_{0}}\left(t_{0}\right)=\phi_{p}\left(t_{0}\right)+\chi\left(t_{0}\right)$ has a unique solution in the interval $[T, \infty)$ such that $\left(w_{t_{0}}, h_{0}^{-1} \varphi_{t_{0}}\right) \in\left(\mathcal{C} \cap L^{\infty}\right)\left([T, \infty), H^{k} \oplus Y^{\ell}\right)$. One can define $T_{0}$ and $T$ by conditions of the type

$$
\begin{gathered}
A\left(a_{+}, b_{+}\right) h\left(T_{0}\right)=1 \\
A\left(a_{+}, b_{+}\right)((p+2) \gamma-1)^{-1} h(T)=1 .
\end{gathered}
$$

The solution satisfies the estimates

$$
\begin{gathered}
\left|w_{t_{0}}(t)-V(t)\right|_{k} \vee\left|w_{t_{0}}(t)-W_{p}(t)\right|_{k} \leq A\left(a_{+}, b_{+}\right) Q_{p}\left(t_{0}\right) \\
\left|\varphi_{t_{0}}(t)-\phi_{p}(t)-\chi(t)\right|_{\ell} \vee\left|\varphi_{t_{0}}(t)-\phi_{p}(t)-\psi_{+}\right|_{\ell} \leq A\left(a_{+}, b_{+}\right) Q_{p}\left(t_{0}\right) h_{0}(t)
\end{gathered}
$$

for $t \geq t_{0}$,

$$
\begin{gathered}
\left|w_{t_{0}}(t)-V(t)\right|_{k} \vee\left|w_{t_{0}}(t)-W_{p}(t)\right|_{k} \leq A\left(a_{+}, b_{+}\right) Q_{p}(t) \\
\left|\varphi_{t_{0}}(t)-\phi_{p}(t)-\chi(t)\right|_{\ell} \vee\left|\varphi_{t_{0}}(t)-\phi_{p}(t)-\psi_{+}\right|_{\ell} \leq A\left(a_{+}, b_{+}\right) P_{p}(t)
\end{gathered}
$$

for $T \leq t \leq t_{0}$, and

$$
\left|w_{t_{0}}(t)\right|_{k} \leq A\left(a_{+}, b_{+}\right) \quad, \quad\left|\varphi_{t_{0}}(t)\right|_{\ell} \leq A\left(a_{+}, b_{+}\right) h_{0}(t)
$$

for all $t \geq T$.

Proof. The result follows from Proposition 4.1 and standard globalisation arguments provided we can derive (6.15) (6.16) (6.17) (6.18) as a priori estimates under the assumptions of the proposition. Let $\left(w_{t_{0}}, \varphi_{t_{0}}\right)$ be the maximal solution of (4.1) (4.2) with the appropriate initial condition at $t_{0}$. Define $\widetilde{q}=w_{t_{0}}-V$ and $\widetilde{\psi}=\varphi_{t_{0}}-\phi_{p}-\chi$. Comparing the equations (4.1) (4.2) and (5.12) (5.39), we obtain

$\partial_{t} \widetilde{q}=\left(2 t^{2}\right)^{-1}\left\{i \Delta w_{t_{0}}+\left(2 \nabla \varphi_{t_{0}} \cdot \nabla+\left(\Delta \varphi_{t_{0}}\right)\right) \widetilde{q}+\left(2 \nabla\left(\widetilde{\psi}+\varphi_{p}+\chi\right) \cdot \nabla+\left(\Delta\left(\widetilde{\psi}+\varphi_{p}+\chi\right)\right)\right) V\right\}$ 


$$
\begin{aligned}
\partial_{t} \widetilde{\psi}= & \left(2 t^{2}\right)^{-1}\left\{|\nabla \widetilde{\psi}|^{2}+2 \nabla \widetilde{\psi} \cdot \nabla\left(\phi_{p}+\chi\right)+|\nabla \chi|^{2}+2 \nabla \chi \cdot \nabla \varphi_{p}+\sum_{\substack{0 \leq i, j \leq p \\
i+j \leq p}} \nabla \varphi_{i} \cdot \nabla \varphi_{j}\right\} \\
& +t^{-\gamma}\left\{g_{0}(\widetilde{q}, \widetilde{q})+2 g_{0}(\widetilde{q}, V)+g_{0}\left(V-W_{p}, V+W_{p}\right)+\sum_{\substack{0 \leq i, j \leq p \\
i+j \geq p+1}} g_{0}\left(w_{i} w_{j}\right)\right\}
\end{aligned}
$$

where the last bracket is obtained by rewriting

$$
g_{0}\left(w_{t_{0}}, w_{t_{0}}\right)-\sum_{i+j \leq p} g_{0}\left(w_{i}, w_{j}\right)
$$

We estimate $(\widetilde{q}, \widetilde{\psi})$ by Lemmas 3.2 and 3.3, especially (3.6) (3.12) for $\widetilde{q}$ and (3.7) (3.8) for $\widetilde{\psi}$ and we obtain

$$
\begin{gathered}
\left.\left|\partial_{t}\right| \widetilde{q}\right|_{k} \mid \leq C t^{-2}\left\{|V|_{k+2}+\left|\varphi_{t_{0}}\right|_{\ell}|\widetilde{q}|_{k}+\left|\widetilde{\psi}+\varphi_{p}+\chi\right|_{\ell}|V|_{k+1}\right\} \\
\left.\left|\partial_{t}\right| \widetilde{\psi}\right|_{\ell} \mid \leq C t^{-2}\left\{|\widetilde{\psi}|_{\ell}^{2}+|\widetilde{\psi}|_{\ell}\left|\phi_{p}+\chi\right|_{\ell+1}+|\chi|_{\ell+1}^{2}+\left|\varphi_{p}\right|_{\ell+1}|\chi|_{\ell+1}+\sum_{\substack{0 \leq i, j \leq p \\
i+j \geq p}}\left|\varphi_{i}\right|_{\ell+1}\left|\varphi_{j}\right|_{\ell+1}\right\} \\
+C t^{-\gamma}\left\{|\widetilde{q}|_{k}^{2}+|\widetilde{q}|_{k}|V|_{k}+\left|V-W_{p}\right|_{k}\left|V+W_{p}\right|_{k}+\sum_{\substack{0 \leq i, j \leq p \\
i+j \geq p+1}}\left|w_{i}\right|_{k}\left|w_{j}\right|_{k}\right\}
\end{gathered}
$$

From Propositions 5.1 and 5.4, it follows that there exist $a$ and $b$ depending on $\left(a_{+}, b_{+}\right)$, such that the following estimates hold.

$$
\begin{aligned}
|V|_{k+2} \leq a \quad, \quad\left|W_{p}\right|_{k} \leq a \quad, \quad\left|V-W_{p}\right|_{k} \leq a Q_{p} \quad, & \\
\left|w_{j}\right|_{k} \leq a Q_{j-1} & \text { for } 1 \leq j \leq p \\
\left|\phi_{p}\right|_{\ell+1} \leq b h_{0} \quad, \quad\left|\varphi_{j}\right|_{\ell+1} \leq b N_{j} & \text { for } 0 \leq j \leq p \\
|\chi|_{\ell+1} \leq b \leq C b N_{p} \leq C b \gamma^{-p} h_{0} & \text { for } t \geq 2 .
\end{aligned}
$$

We now define $y=|\widetilde{q}|_{k}$ and $z=|\widetilde{\psi}|_{\ell}$. Using the previous estimates, we obtain from (6.22) $(6.23)$

$$
\begin{gathered}
\left|\partial_{t} y\right| \leq C t^{-2}\left\{a+\left(z+b h_{0}\right) y+\left(z+b N_{p}\right) a\right\} \\
\left|\partial_{t} z\right| \leq C t^{-2}\left\{\left(z+b h_{0}\right) z+b^{2} \sum_{\substack{0 \leq i, j \leq p \\
i+j \geq p}} N_{i} N_{j}\right\}+C t^{-\gamma}\left\{y(y+a)+a^{2} Q_{p}+a^{2} \sum_{\substack{0 \leq i, j \leq p-1 \\
i+j \geq p-1}} Q_{i} Q_{j}\right\} .
\end{gathered}
$$

In the last bracket in (6.25), the terms in $a^{2}$ are absent for $p=0$ since in that case $V=W_{0}=$ $w_{0}=w_{+}$.

We next estimate $y$ and $z$ from (6.24) (6.25), taking $C=1$ for the rest of the proof. We distinguish two cases. 
Case $\mathbf{t} \geq \mathbf{t}_{\mathbf{0}}$. Let $\bar{t}>t_{0}$ and define $Y=Y(\bar{t})=\left\|y ; L^{\infty}\left(\left[t_{0}, \bar{t}\right]\right)\right\|$ and $Z=Z(\bar{t})=$ $\left\|h_{0}^{-1} z ; L^{\infty}\left(\left[t_{0}, \bar{t}\right]\right)\right\|$. Then for all $t \in\left[t_{0}, \bar{t}\right]$

$$
\begin{gathered}
\partial_{t} y \leq t^{-2}\left\{a+(Z+b) Y h_{0}+a Z h_{0}+a b N_{p}\right\} \\
\partial_{t} z \leq t^{-2}\left\{(Z+b) Z h_{0}^{2}+b^{2} \sum_{\substack{0 \leq i, j \leq p \\
i+j \geq p}} N_{i} N_{j}\right\}+t^{-\gamma}\left\{Y(Y+a)+a^{2} Q_{p}+a^{2} \sum_{\substack{0 \leq j \leq j \leq p-1 \\
i+j \geq p-1}} Q_{i} Q_{j}\right\} .
\end{gathered}
$$

Integrating (6.26) between $t_{0}$ and $t$ and using (3.35) we obtain

$$
y \leq a t_{0}^{-1}+(Z+b) Y h\left(t_{0}\right)+a Z h\left(t_{0}\right)+a b Q_{p}\left(t_{0}\right)
$$

and therefore

$$
Y \leq(Z+b) Y h\left(t_{0}\right)+a Z h\left(t_{0}\right)+a B_{1}\left(t_{0}\right)
$$

where

$$
B_{1}\left(t_{0}\right)=t_{0}^{-1}+b Q_{p}\left(t_{0}\right) \leq\left(Q_{p}(1)^{-1}+b\right) Q_{p}\left(t_{0}\right) \quad .
$$

Integrating (6.27) between $t_{0}$ and $t$, we obtain similarly

$$
z \leq(Z+b) Z h_{0}(t) h\left(t_{0}\right)+Y(Y+a) h_{0}(t)+C\left(b^{2}+a^{2}\right) h_{0}(t) Q_{p}\left(t_{0}\right)
$$

Here we have used the following relations

$$
\begin{gathered}
\int_{t_{0}}^{t} d t_{1} t_{1}^{-2} h_{0}^{2}\left(t_{1}\right) \leq h_{0}(t)\left(h\left(t_{0}\right)-h(t)\right) \\
\int_{t_{0}}^{t} d t_{1} t_{1}^{-2} N_{i}\left(t_{1}\right) N_{j}\left(t_{1}\right) \leq \int_{t_{0}}^{t} d t_{1} t_{1}^{-2} h_{0}\left(t_{1}\right) N_{i+j}\left(t_{1}\right) \\
\leq h_{0}(t) \int_{t_{0}}^{t} d t_{1} t_{1}^{-2} N_{i+j}\left(t_{1}\right)=h_{0}(t)\left(Q_{i+j}\left(t_{0}\right)-Q_{i+j}(t)\right)
\end{gathered}
$$

by (3.38) (3.35),

$$
\int_{t_{0}}^{t} d t_{1} t_{1}^{-\gamma} Q_{p}\left(t_{1}\right) \leq Q_{p}\left(t_{0}\right)\left(h_{0}(t)-h_{0}\left(t_{0}\right)\right)
$$

by (3.45), and

$$
\int_{t_{0}}^{t} d t_{1} t_{1}^{-\gamma} Q_{i}\left(t_{1}\right) Q_{j}\left(t_{1}\right) \leq \int_{t_{0}}^{t} d t_{1} t_{1}^{-\gamma} h\left(t_{1}\right) Q_{i+j}\left(t_{1}\right) \leq 2 Q_{i+j+1}\left(t_{0}\right)\left(h_{0}(t)-h_{0}\left(t_{0}\right)\right)
$$

by (3.40) and (3.46).

¿From (6.31) we obtain

$$
Z \leq(Z+b) Z h\left(t_{0}\right)+Y(Y+a)+B_{2}\left(t_{0}\right)
$$


with

$$
B_{2}=C\left(b^{2}+a^{2}\right) Q_{p}\left(t_{0}\right)
$$

Now (6.29) (6.32) define a closed subset $\mathcal{R}$ of $I R^{+} \times I R^{+}$in the $(Y, Z)$ variables, containing the point $(0,0)$, and $(Y, Z)$ is a continuous function of $\bar{t}$ starting from that point for $\bar{t}=t_{0}$. If we can find an open region $\mathcal{R}_{1}$ of $I^{+} \times \mathbb{R}^{+}$containing $(0,0)$ and such that $\overline{\mathcal{R} \cap \mathcal{R}_{1}} \subset \mathcal{R}_{1}$, then $(Y, Z)$ will remain in $\mathcal{R} \cap \mathcal{R}_{1}$ for all time, because $\overline{\mathcal{R} \cap \mathcal{R}_{1}}$ is both open and closed in $\mathcal{R}$. We first take $t_{0}$ sufficiently large so that

$$
4 b h\left(t_{0}\right) \leq 1 \quad, \quad 16 a^{2} h\left(t_{0}\right) \leq 1 \quad, \quad 4 B_{1}\left(t_{0}\right) \leq 1
$$

and we choose for $\mathcal{R}_{1}$ the region $4 Z h\left(t_{0}\right)<1$. From (6.29) (6.32) (6.34) it follows that in $\overline{\mathcal{R} \cap \mathcal{R}_{1}}$

$$
\left\{\begin{array}{l}
Y \leq 2 a\left(Z h\left(t_{0}\right)+B_{1}\left(t_{0}\right)\right) \leq a \\
Z \leq 4 a Y+2 B_{2}\left(t_{0}\right)
\end{array}\right.
$$

and therefore

$$
\left\{\begin{array}{l}
Y \leq 4 a B_{1}\left(t_{0}\right)+2 B_{2}\left(t_{0}\right) h\left(t_{0}\right) \\
Z \leq 4\left(4 a^{2} B_{1}\left(t_{0}\right)+B_{2}\left(t_{0}\right)\right)
\end{array}\right.
$$

so that the condition $4 Z h\left(t_{0}\right)<1$ is implied by

$$
16\left(4 a^{2} B_{1}\left(t_{0}\right)+B_{2}\left(t_{0}\right)\right) h\left(t_{0}\right)<1 .
$$

The estimates (6.15) (6.16) with $V$ and $\chi$ now follow from (6.35), while the conditions (6.34) (6.36) reduce to the form (6.13).

The estimates (6.15) (6.16) with $W_{p}$ and $\psi_{+}$follow from the previous ones, from (5.43) (5.44) and from the fact that

$$
Q_{p}(t) h_{0}(t) h(t)^{-1}=\left(t Q_{p}(t)\right)\left(h_{0}(t) t^{-1} h(t)^{-1}\right)
$$

is an increasing function of $t$, so that

$$
h(t) \leq h\left(t_{1}\right) Q_{p}\left(t_{1}\right)^{-1} h_{0}\left(t_{1}\right)^{-1} Q_{p}\left(t_{0}\right) h_{0}(t)
$$

for any (fixed) $t_{1} \leq t_{0}$. 
Case $\mathbf{t} \leq \mathbf{t}_{\mathbf{0}}$. Let $\bar{t}<t_{0}$ and define $Y=Y(\bar{t})=\left\|Q_{p}^{-1} y ; L^{\infty}\left(\left[\bar{t}, t_{0}\right]\right)\right\|$ and $Z=Z(\bar{t})=$ $\left\|P_{p}^{-1} z ; L^{\infty}\left(\left[\bar{t}, t_{0}\right]\right)\right\|$. It then follows from (6.24) (6.25) that for all $t \in\left[\bar{t}, t_{0}\right]$

$$
\begin{gathered}
\left|\partial_{t} y\right| \leq t^{-2}\left\{a+Z Y P_{p} Q_{p}+b Y h_{0} Q_{p}+a Z P_{p}+a b N_{p}\right\} \\
\left|\partial_{t} z\right| \leq t^{-2}\left\{Z^{2} P_{p}^{2}+b Z h_{0} P_{p}+b^{2} \sum_{\substack{0 \leq i, j \leq p \\
i+j \geq p}} N_{i} N_{j}\right\} \\
+t^{-\gamma}\left\{Y^{2} Q_{p}^{2}+a Y Q_{p}+a^{2} Q_{p}+a^{2} \sum_{\substack{0 \leq i, j \leq p-1 \\
i+j \geq p-1}} Q_{i} Q_{j}\right\} .
\end{gathered}
$$

Integrating (6.37) between $t$ and $t_{0}$, using (3.32) (3.35) and

$$
\begin{aligned}
& \int_{t}^{t_{0}} d t_{1} t_{1}^{-2} P_{p}\left(t_{1}\right) Q_{p}\left(t_{1}\right) \leq Q_{p}(t)\left(R_{p}(t)-R_{p}\left(t_{0}\right)\right) \\
& \int_{t}^{t_{0}} d t_{1} t_{1}^{-2} h_{0}\left(t_{1}\right) Q_{p}\left(t_{1}\right) \leq Q_{p}(t)\left(h(t)-h\left(t_{0}\right)\right)
\end{aligned}
$$

we obtain

$$
y \leq a t^{-1}+Z Y R_{p}(t) Q_{p}(t)+b Y h(t) Q_{p}(t)+a Z R_{p}(t)+a b Q_{p}(t)
$$

and therefore by $(3.47)$

$$
Y \leq a c+Z Y R_{p}(t)+b Y h(t)+C_{p} a Z h(t)+a b
$$

where $c=Q_{p}(1)^{-1}$.

We integrate similarly (6.38) between $t$ and $t_{0}$. We use the relations

$$
\begin{aligned}
\int_{t}^{t_{0}} d t_{1} t_{1}^{-2} P_{p}^{2}\left(t_{1}\right) & \leq P_{p}(t)\left(R_{p}(t)-R_{p}\left(t_{0}\right)\right) \leq P_{p}(t) R_{p}(t) \\
\int_{t}^{t_{0}} d t_{1} t_{1}^{-2} h_{0}\left(t_{1}\right) P_{p}\left(t_{1}\right) & \leq P_{p}(t)\left(h(t)-h\left(t_{0}\right)\right) \\
\int_{t}^{t_{0}} d t_{1} t_{1}^{-2} N_{i}\left(t_{1}\right) N_{j}\left(t_{1}\right) & \leq \int_{t}^{t_{0}} d t_{1} t_{1}^{-2} h_{0}\left(t_{1}\right) N_{i+j}\left(t_{1}\right) \\
& \leq P_{i+j}(t)-P_{i+j}\left(t_{0}\right)
\end{aligned}
$$

by (3.38) (3.37),

$$
\int_{t}^{t_{0}} d t_{1} t_{1}^{-\gamma} Q_{p}^{2}\left(t_{1}\right) \leq Q_{p}(t) \int_{t}^{t_{0}} d t_{1} t_{1}^{-\gamma} Q_{p}\left(t_{1}\right) \leq Q_{p}(t) P_{p}(t)
$$

by (3.42) and

$$
\int_{t}^{t_{0}} d t_{1} t_{1}^{-\gamma} Q_{i}\left(t_{1}\right) Q_{j}\left(t_{1}\right) \leq P_{i+j+1}(t)
$$


by $(3.40)(3.41)(3.42)$. We obtain

$$
z \leq Z^{2} P_{p}(t) R_{p}(t)+b Z P_{p}(t) h(t)+Y^{2} P_{p}(t) Q_{p}(t)+a Y P_{p}(t)+C\left(a^{2}+b^{2}\right) P_{p}(t)
$$

and therefore

$$
Z \leq Z^{2} R_{p}(t)+b Z h(t)+Y^{2} Q_{p}(t)+a Y+C\left(a^{2}+b^{2}\right)
$$

We now take $t$ sufficiently large so that $b h(t) \leq 1 / 4$ and we proceed as in the case $t \geq t_{0}$ by taking for $\mathcal{R}_{1}$ the strip defined by $Z R_{p}(t)<1 / 4, C_{p} Z h(t)<b$, thereby obtaining from (6.40) $(6.42)$

$$
\left\{\begin{array}{l}
Y \leq 2 a(2 b+c) \\
Z \leq 2 C\left(a^{2}+b^{2}\right)+4 a^{2}(2 b+c)\left(1+2(2 b+c) Q_{p}(1)\right)
\end{array}\right.
$$

The conditions $b h \leq 1 / 4, Z R<1 / 4, Z h<b$ are then satisfied for $t \geq T$ with $T$ defined by a condition of the form $(6.14)$, where the singular factor $((p+2) \gamma-1)^{-1}$ comes from $C_{p}$. The estimates $(6.17)(6.18)$ with $V$ and $\chi$ follow from (6.43), and the analogous estimates with $W_{p}$ and $\psi_{+}$follow therefrom and from (5.43) (5.44).

Finally the estimates (6.19) follow from (6.15) (6.16) (6.17) (6.18) and from Proposition 5.1.

We can now take the limit $t_{0} \rightarrow \infty$ of the solution $\left(w_{t_{0}}, \varphi_{t_{0}}\right)$ constructed in Proposition 6.2, for fixed $\left(w_{+}, \psi_{+}\right)$.

Proposition 6.3. Let $(k, \ell)$ be an admissible pair and let $p$ be an integer such that $(p+2) \gamma>1$. Let $w_{+} \in H^{k+(p+1) \vee 2}$ and $\psi_{+} \in Y^{\ell+1}$. Let $\phi=\phi_{p-1}$ be defined by (5.11) and Proposition 5.1, so that $h_{0}^{-1} \phi \in\left(\mathcal{C} \cap L^{\infty}\right)\left([1, \infty), Y^{\ell+2}\right)$. Let $V$ and $\chi$ be the solutions of (5.12) and (5.39) respectively, obtained in Proposition 5.4 so that $(V, \chi) \in\left(\mathcal{C} \cap L^{\infty}\right)\left([1, \infty), H^{k+2} \oplus Y^{\ell+1}\right)$ and let $a_{+}, b_{+}$be defined by (6.12). Then

(1) There exists $T, 1 \leq T<\infty$, depending only on $\left(\gamma, p, a_{+}, b_{+}\right)$and there exists a unique solution $(w, \varphi)$ of the system (4.1) (4.2) in the interval $[T, \infty)$ such that $\left(w, h_{0}^{-1} \varphi\right) \in(\mathcal{C} \cap$ $\left.L^{\infty}\right)\left([T, \infty), H^{k} \oplus Y^{\ell}\right)$ and such that the following estimates hold for all $t \geq T$.

$$
|w(t)-V(t)|_{k} \vee\left|w(t)-W_{p}(t)\right|_{k} \leq A\left(a_{+}, b_{+}\right) Q_{p}(t)
$$




$$
\begin{gathered}
\left|\varphi(t)-\phi_{p}(t)-\chi(t)\right|_{\ell} \vee\left|\varphi(t)-\phi_{p}(t)-\psi_{+}\right|_{\ell} \leq A\left(a_{+}, b_{+}\right) P_{p}(t) \\
|w(t)|_{k} \leq A\left(a_{+}, b_{+}\right) \quad, \quad|\varphi(t)|_{\ell} \leq A\left(a_{+}, b_{+}\right) h_{0}(t)
\end{gathered}
$$

One can define $T$ by a condition of the type (6.14).

(2) Let $\left(w_{t_{0}}, \varphi_{t_{0}}\right)$ be the solution of the system (4.1) (4.2) constructed in Proposition 6.2 for $t_{0} \geq T_{0} \vee T$ and such that $\left(w_{t_{0}}, h_{0}^{-1} \varphi_{t_{0}}\right) \in\left(\mathcal{C} \cap L^{\infty}\right)\left([T, \infty), H^{k} \oplus Y^{\ell}\right)$. Then $\left(w_{t_{0}}, \varphi_{t_{0}}\right)$ converges to $(w, \varphi)$ in norm in $L^{\infty}\left(J, H^{k-1} \oplus Y^{\ell-1}\right)$ and in the weak-* sense in $L^{\infty}\left(J, H^{k} \oplus Y^{\ell}\right)$ for any compact $J \subset[T, \infty)$, and in the weak-* sense in $H^{k} \oplus Y^{\ell}$ pointwise in $t$.

(3) The map $\left(w_{+}, \psi_{+}\right) \rightarrow(w, \varphi)$ defined in Part (1) is continuous on the bounded sets of $H^{k+(p+1) \vee 2} \oplus Y^{\ell+1}$ from the norm topology of $\left(w_{+}, \psi_{+}\right)$in $H^{k+p-1} \oplus Y^{\ell-1}$ to the norm topology of $(w, \varphi)$ in $L^{\infty}\left(J, H^{k-1} \oplus Y^{\ell-1}\right)$ and to the weak-* topology in $L^{\infty}\left(J, H^{k} \oplus Y^{\ell}\right)$ for any compact interval $J \subset[T, \infty)$, and to the weak-* topology in $H^{k} \oplus Y^{\ell}$ pointwise in $t$.

Proof. Parts (1) and (2) will follow from the convergence of $\left(w_{t_{0}}, \varphi_{t_{0}}\right)$ when $t_{0} \rightarrow \infty$ in the topologies stated in Part (2). Let $T_{0} \vee T \leq t_{0} \leq t_{1}$. From (6.17) (6.18) it follows that

$$
\begin{gathered}
\left|w_{t_{1}}\left(t_{0}\right)-w_{t_{0}}\left(t_{0}\right)\right|_{k}=\left|w_{t_{1}}\left(t_{0}\right)-V\left(t_{0}\right)\right|_{k} \leq A Q_{p}\left(t_{0}\right) \\
\left|\varphi_{t_{1}}\left(t_{0}\right)-\varphi_{t_{0}}\left(t_{0}\right)\right|_{\ell}=\left|\varphi_{t_{1}}\left(t_{0}\right)-\phi_{p}\left(t_{0}\right)-\chi\left(t_{0}\right)\right|_{\ell} \leq A P_{p}\left(t_{0}\right)
\end{gathered}
$$

We now estimate $\left(w_{t_{0}}-w_{t_{1}}, \varphi_{t_{0}}-\varphi_{t_{1}}\right)$ in $H^{k-1} \oplus Y^{\ell-1}$ for $t \leq t_{0}$. Let

$$
y=\left|w_{t_{0}}-w_{t_{1}}\right|_{k-1} \quad, \quad z=\left|\varphi_{t_{0}}-\varphi_{t_{1}}\right|_{\ell-1}
$$

From (6.19) and Lemma 3.3, it follows that $y$ and $z$ satisfy the system (4.10). Integrating that system for $t \leq t_{0}$ with initial data at $t_{0}$, we obtain from Lemma 4.1

$$
\left\{\begin{array}{l}
y(t) \leq A\left(y\left(t_{0}\right)+t^{-1}\left(z\left(t_{0}\right)+y\left(t_{0}\right) h_{0}\left(t_{0}\right)\right)\right) \\
z(t) \leq A\left(z\left(t_{0}\right)+y\left(t_{0}\right) h_{0}\left(t_{0}\right)\right)
\end{array}\right.
$$

From (6.47) (6.48) (6.50) and from the fact that $P_{p}\left(t_{0}\right)$ and $Q_{p}\left(t_{0}\right) h_{0}\left(t_{0}\right)$ tend to zero when $t_{0} \rightarrow \infty$, it follows that there exists $(w, \varphi) \in \mathcal{C}\left([T, \infty), H^{k-1} \oplus Y^{\ell-1}\right)$ such that $\left(w_{t_{0}}, \varphi_{t_{0}}\right)$ converges to $(w, \varphi)$ in $L^{\infty}\left(J, H^{k-1} \oplus Y^{\ell-1}\right)$ for all compact intervals $J \subset[T, \infty)$. ¿From that convergence, from (6.17) (6.18) (6.19) and standard compactness arguments, it follows that 
$\left(w, h_{0}^{-1} \varphi\right) \in\left(\mathcal{C}_{w *} \cap L^{\infty}\right)\left([T, \infty), H^{k} \oplus Y^{\ell}\right)$, that $(w, \varphi)$ satisfies the estimates (6.44) (6.45) (6.46) for all $t \geq T$, and that $\left(w_{t_{0}}, \varphi_{t_{0}}\right)$ converges to $(w, \varphi)$ in the other topologies considered in Part (2). Furthermore, $(w, \varphi)$ satisfies the system (4.1) (4.2) and by Proposition 4.1, part (1), $(w, \varphi) \in \mathcal{C}\left([T, \infty), H^{k} \oplus Y^{\ell}\right)$. Finally, uniqueness of $(w, \varphi)$ under the conditions (6.44) (6.45) follows from Proposition 4.3 and from the fact that $P_{p}(t)$ and $Q_{p}(t) h_{0}(t)$ tend to zero when $t \rightarrow \infty$.

Part (3). Let $\left(w_{+}, \psi_{+}\right)$and $\left(w_{+}^{\prime}, \psi_{+}^{\prime}\right)$ belong to a fixed bounded set of $H^{k+(p+1) \vee 2} \oplus Y^{\ell+1}$. Let $\left(W_{p}, \phi_{p}\right)$ and $\left(W_{p}^{\prime}, \phi_{p}^{\prime}\right)$ be the associated functions defined by (5.11) and Proposition 5.1 and let $(w, \varphi)$ and $\left(w^{\prime}, \varphi^{\prime}\right)$ be the associated solutions of the system (4.1) (4.2) defined in Part (1). We assume that $\left(w_{+}^{\prime}, \psi_{+}^{\prime}\right)$ is close to $\left(w_{+}, \psi_{+}\right)$in the sense that

$$
\begin{aligned}
& \left|w_{+}-w_{+}^{\prime}\right|_{k+p-1} \leq \varepsilon \\
& \left|\psi_{+}-\psi_{+}^{\prime}\right|_{\ell-1} \leq \varepsilon_{0} .
\end{aligned}
$$

We now take $t_{0}>T$ and we estimate $\left(w-w^{\prime}, \varphi-\varphi^{\prime}\right)$ in $H^{k-1} \oplus Y^{\ell-1}$ for $t \leq t_{0}$. Let

$$
y=\left|w-w^{\prime}\right|_{k-1} \quad, \quad z=\left|\varphi-\varphi^{\prime}\right|_{\ell-1}
$$

From (4.1) (4.2) and Lemma 3.3, it follows that $(y, z)$ satisfy the system (4.10). Integrating that system between $t_{0}$ and $t$ yields the estimate (6.50) for $(y, z)$ defined by (6.53). From (6.44) (6.45) we obtain

$$
\left\{\begin{array}{l}
y\left(t_{0}\right) \leq A Q_{p}\left(t_{0}\right)+\left|W_{p}\left(t_{0}\right)-W_{p}^{\prime}\left(t_{0}\right)\right|_{k-1} \\
z\left(t_{0}\right) \leq A P_{p}\left(t_{0}\right)+\varepsilon_{0}+\left|\phi_{p}\left(t_{0}\right)-\phi_{p}^{\prime}\left(t_{0}\right)\right|_{\ell-1}
\end{array} .\right.
$$

From estimates similar to (5.8) (5.9), and from (6.51) (6.52) it then follows that

$$
\left\{\begin{array}{l}
y\left(t_{0}\right) \leq A\left(Q_{p}\left(t_{0}\right)+\varepsilon\right) \\
z\left(t_{0}\right) \leq A\left(P_{p}\left(t_{0}\right)+\varepsilon h_{0}\left(t_{0}\right)\right)+\varepsilon_{0} .
\end{array}\right.
$$

We now choose $t_{0}$ so that $Q_{p}\left(t_{0}\right)=\varepsilon$. Substituting (6.55) with that choice into (6.50) and using the asymptotic behaviour of $Q_{p}$ and $P_{p}$ for large $t$, we obtain

$$
\left\{\begin{array}{l}
y(t) \leq A\left(m(\varepsilon)+t^{-1}\left(\varepsilon_{0}+m(\varepsilon)\right)\right) \\
z(t) \leq A\left(\varepsilon_{0}+m(\varepsilon)\right)
\end{array}\right.
$$


where

$$
m(\varepsilon)= \begin{cases}\varepsilon^{((p+2) \gamma-1) /(p+1) \gamma} & \text { for }(p+1) \gamma<1 \\ \varepsilon^{\gamma} \log \varepsilon & \text { for }(p+1) \gamma=1 \\ \varepsilon^{\gamma} & \text { for }(p+1) \gamma>1 .\end{cases}
$$

This implies the (uniform Hölder) continuity of $(w, \varphi)$ as a function of $\left(w_{+}, \psi_{+}\right)$in the norm topology of $L^{\infty}\left(J, H^{k-1} \oplus Y^{\ell-1}\right)$ for all compact intervals $J \subset[T, \infty)$. The other continuities follow therefrom and from the boundedness of $\left(w, h_{0}^{-1} \varphi\right)$ in $L^{\infty}\left([T, \infty), H^{k} \oplus Y^{\ell}\right)$ by standard compactness arguments.

\section{Asymptotics and wave operators for $u$}

In this section we complete the construction of the wave operators for the equation (1.1) and we derive asymptotic properties of solutions in their range. The construction relies in an essential way on those of Section 6, esp. Proposition 6.3, and will require a discussion of the gauge invariance of those constructions.

We first define the wave operator for the auxiliary system (4.1) (4.2).

Definition 7.1. We define the wave operator $\Omega_{0}$ as the map

$$
\Omega_{0}:\left(w_{+}, \psi_{+}\right) \rightarrow(w, \varphi)
$$

from $H^{k+(p+1) \vee 2} \oplus Y^{\ell+1}$ to the space of $(w, \varphi)$ such that $\left(w, h_{0}^{-1} \varphi\right) \in\left(\mathcal{C} \cap L^{\infty}\right)\left([T, \infty), H^{k} \oplus Y^{\ell}\right)$ for some $T, 1 \leq T<\infty$, where $(w, \varphi)$ is the solution of the system (4.1) (4.2) obtained in Proposition 6.3, part (1).

Before defining the wave operators for $u$, we now study the gauge invariance of $\Omega_{0}$, which plays an important role in justifying that definition, as was explained in Section 2. For that purpose we need some information on the Cauchy problem for the equation (1.1) at finite times. In addition to the operators $M=M(t)$ and $D=D(t)$ defined by (2.4) (2.5), we introduce the operator

$$
J=J(t)=x+i t \nabla
$$


the generator of Galilei transformations. The operators $M, D, J$ satisfy the commutation relation

$$
i M D \nabla=J M D \text {. }
$$

For any interval $I \subset[1, \infty)$ and any nonnegative integer $k$, we define the space

$$
\begin{aligned}
\mathcal{X}^{k}(I) & =\left\{u: D^{*} M^{*} u \in \mathcal{C}\left(I, H^{k}\right)\right\} \\
& =\left\{u:<J(t)>^{k} u \in \mathcal{C}\left(I, L^{2}\right)\right\}
\end{aligned}
$$

where $\langle\lambda\rangle=\left(1+\lambda^{2}\right)^{1 / 2}$ for any real number or self-adjoint operator $\lambda$ and where the second equality follows from (7.3). Then [3]

Proposition 7.1. Let $k$ be a positive integer and let $0<\mu<n \wedge 2 k$. Then the Cauchy problem for the equation (1.1) with initial data $u\left(t_{0}\right)=u_{0}$ such that $<J\left(t_{0}\right)>^{k} u_{0} \in L^{2}$ at some initial time $t_{0} \geq 1$ is locally well posed in $\mathcal{X}^{k}(\cdot)$, namely

(1) There exists $T>0$ such that (1.1) has a unique solution with initial data $u\left(t_{0}\right)=u_{0}$ in $\mathcal{X}^{k}\left(\left[1 \vee\left(t_{0}-T\right), t_{0}+T\right]\right)$.

(2) For any interval $I, t_{0} \in I \subset[1, \infty)$, (1.1) with initial data $u\left(t_{0}\right)=u_{0}$ has at most one solution in $\mathcal{X}^{k}(I)$.

(3) The solution of Part (1) depends continuously on $u_{0}$ in the norms considered there.

We come back from the system (4.1) (4.2) to the equation (1.1) by reconstructing $u$ from $(w, \varphi)$ by $(2.7)$ and accordingly we define the map

$$
\Lambda:(w, \varphi) \rightarrow u=M D \exp (-i \varphi) w
$$

It follows immediately from Lemma 3.1 that the map $\Lambda$ satisfies the following property.

Lemma 7.1. The map $\Lambda$ defined by (7.5) is bounded and continuous from $\mathcal{C}\left(I, H^{k} \oplus Y^{\ell}\right)$ to $\mathcal{X}^{k}(I)$ for any admissible pair $(k, \ell)$ and any interval $I \subset[1, \infty)$.

We now give the following definition. 
Definition 7.2. Let $(k, \ell)$ be an admissible pair and let $(w, \varphi)$ and $\left(w^{\prime}, \varphi^{\prime}\right)$ be two solutions of the system (4.1) (4.2) in $\mathcal{C}\left(I, H^{k} \oplus Y^{\ell}\right)$ for some interval $I \subset[1, \infty)$. We say that $(w, \varphi)$ and $\left(w^{\prime}, \varphi^{\prime}\right)$ are gauge equivalent if they give rise to the same $u$, namely if $\Lambda(w, \varphi)=\Lambda\left(w^{\prime}, \varphi^{\prime}\right)$, or equivalently if

$$
\exp (-i \varphi(t)) w(t)=\exp \left(-i \varphi^{\prime}(t)\right) w^{\prime}(t)
$$

for all $t \in I$.

A sufficient condition for gauge equivalence is given by the following Lemma.

Lemma 7.2. Let $(k, \ell)$ be an admissible pair and let $(w, \varphi)$ and $\left(w^{\prime}, \varphi^{\prime}\right)$ be two solutions of the system (4.1) (4.2) in $\mathcal{C}\left(I, H^{k} \oplus Y^{\ell}\right)$. In order that $(w, \varphi)$ and $\left(w^{\prime}, \varphi^{\prime}\right)$ be gauge equivalent, it is sufficient that (7.6) holds for one $t \in I$.

Proof. An immediate consequence of Lemma 7.1, of Proposition 7.1, part (2), and of the fact that $(k, \ell)$ admissible implies $k>1+\mu / 2$.

The gauge covariance properties of $\Omega_{0}$ will be expressed by the following two propositions.

Proposition 7.2. Let $(k, \ell)$ be an admissible pair. Let $(w, \varphi)$ and $\left(w^{\prime}, \varphi^{\prime}\right)$ be two solutions of the system (4.1) (4.2) such that $\left(w, h_{0}^{-1} \varphi\right),\left(w^{\prime}, h_{0}^{-1} \varphi^{\prime}\right) \in\left(\mathcal{C} \cap L^{\infty}\right)\left([T, \infty), H^{k} \oplus Y^{\ell}\right)$ for some $T \geq 1$, and assume that $(w, \varphi)$ and $\left(w^{\prime}, \varphi^{\prime}\right)$ are gauge equivalent. Then

(1) There exists $\sigma \in Y^{\ell-1}$ such that $\varphi^{\prime}(t)-\varphi(t)$ converges to $\sigma$ when $t \rightarrow \infty$ strongly in $Y^{\ell-2}$ and in the weak-* sense in $Y^{\ell-1}$. The following estimates hold:

$$
\left\|\varphi^{\prime}(t)-\varphi(t)-\sigma ; Y^{\ell-2}\right\| \leq A h(t)
$$

for some constant $A$ depending on $T$ and on the norms of $h_{0}^{-1} \varphi, h_{0}^{-1} \varphi^{\prime}$ in $L^{\infty}\left(\cdot, Y^{\ell}\right)$, with the exception of the case $n$ even, $\ell=n / 2+1$ where the $L^{\infty}$ norm of $\nabla \varphi$ satisfies only

$$
\left\|\nabla \varphi^{\prime}(t)-\nabla \varphi(t)-\nabla \sigma\right\|_{\infty} \leq A h(t)^{1 / 2}
$$

(2) Let $w_{+}$and $w_{+}^{\prime}$ be the limits of $w(t)$ and $w^{\prime}(t)$ as $t \rightarrow \infty$, obtained in Proposition 4.4 . Then $w_{+}^{\prime}=w_{+} \exp (-i \sigma)$. 
(3) Let $p \geq 0$ be an integer. Assume in addition that $w_{+}, w_{+}^{\prime} \in H^{k+p}$ and let $\phi_{p}, \phi_{p}^{\prime}$ be associated with $w_{+}, w_{+}^{\prime}$ according to (5.11) and Proposition 5.1. Assume that the following limits exist

$$
\lim _{t \rightarrow \infty}\left(\varphi(t)-\phi_{p}(t)\right)=\psi_{+} \quad, \quad \lim _{t \rightarrow \infty}\left(\varphi^{\prime}(t)-\phi_{p}^{\prime}(t)\right)=\psi_{+}^{\prime}
$$

as strong limits in $L^{\infty}$. Then $\psi_{+}^{\prime}=\psi_{+}+\sigma$.

Proof. Part (1) is essentially identical with Proposition I.7.2, part (1).

Part (2). We define $\varphi_{-}(t)=\varphi^{\prime}(t)-\varphi(t)$ and we estimate

$$
\begin{gathered}
\left\|w_{+}^{\prime}-w_{+} e^{i \sigma}\right\|_{2} \leq\left\|w_{+}^{\prime}-w^{\prime}(t)\right\|_{2}+\left\|w^{\prime}(t)-\exp \left(i \varphi_{-}(t)\right) w(t)\right\|_{2} \\
\quad+\left\|\left(\exp \left(i \varphi_{-}(t)\right)-\exp (i \sigma)\right) w(t)\right\|_{2}+\left\|\exp (i \sigma)\left(w(t)-w_{+}\right)\right\|_{2} \\
\leq\left\|w_{+}^{\prime}-w^{\prime}(t)\right\|_{2}+\left\|w(t)-w_{+}\right\|_{2}+\left\|\varphi_{-}(t)-\sigma\right\|_{\infty}\|w(t)\|_{2}
\end{gathered}
$$

by gauge invariance. The last member of (7.10) tends to zero as $t \rightarrow \infty$.

Part (3). By gauge invariance, namely Proposition 5.1 part (2) and Part (2) of this proposition, $\phi_{p}^{\prime}=\phi_{p}$ and therefore

$$
\psi_{+}^{\prime}-\psi_{+}=\lim _{t \rightarrow \infty}\left(\varphi^{\prime}(t)-\varphi(t)\right)=\sigma
$$

Remark 7.1. The additional assumptions of Proposition 7.2, part (3) are satisfied either if $(w, \varphi),\left(w^{\prime}, \varphi^{\prime}\right)$ satisfy the assumptions of Proposition 6.1 , or if $(w, \varphi),\left(w^{\prime}, \varphi^{\prime}\right) \in \mathcal{R}\left(\Omega_{0}\right)$. We shall not consider the former case any further. In the latter case, it follows from (7.11) that actually $\sigma \in Y^{\ell+1}$.

Proposition 7.2 prompts us to make the following definition of gauge equivalence for asymptotic states.

Definition 7.3. Two pairs $\left(w_{+}, \psi_{+}\right)$and $\left(w_{+}^{\prime}, \psi_{+}^{\prime}\right)$ are gauge equivalent if $w_{+} \exp \left(-i \psi_{+}\right)=$ $w_{+}^{\prime} \exp \left(-i \psi_{+}^{\prime}\right)$. 
With this definition, Proposition 7.2 implies that two gauge equivalent solutions of the system (4.1) (4.2) in $\mathcal{R}\left(\Omega_{0}\right)$ are images of two gauge equivalent pairs of asymptotic states. The next proposition shows that conversely two gauge equivalent pairs of asymptotic states have gauge equivalent images under $\Omega_{0}$.

Proposition 7.3. Let $(k, \ell)$ be an admissible pair and let $p$ be an integer such that $(p+2) \gamma>1$. Let $\left(w_{+}, \psi_{+}\right),\left(w_{+}^{\prime}, \psi_{+}^{\prime}\right) \in H^{k+(p+1) \vee 2} \oplus Y^{\ell+1}$ be gauge equivalent, and let $(w, \varphi),\left(w^{\prime}, \varphi^{\prime}\right)$ be their images under $\Omega_{0}$. Then $(w, \varphi)$ and $\left(w^{\prime}, \varphi^{\prime}\right)$ are gauge equivalent.

Proof. Let $t_{0}$ be sufficiently large and let $\left(w_{t_{0}}, \varphi_{t_{0}}\right)$ and $\left(w_{t_{0}}^{\prime}, \varphi_{t_{0}}^{\prime}\right)$ be the solutions of the system (4.1) (4.2) constructed by Proposition 6.2. From the initial conditions

$$
\begin{gathered}
w_{t_{0}}\left(t_{0}\right)=V\left(t_{0}\right) \quad, \quad w_{t_{0}}^{\prime}\left(t_{0}\right)=V^{\prime}\left(t_{0}\right), \\
\varphi_{t_{0}}\left(t_{0}\right)=\phi_{p}\left(t_{0}\right)+\chi\left(t_{0}\right) \quad, \quad \varphi_{t_{0}}^{\prime}\left(t_{0}\right)=\phi_{p}^{\prime}\left(t_{0}\right)+\chi^{\prime}\left(t_{0}\right),
\end{gathered}
$$

from the fact that $\phi_{p}=\phi_{p}^{\prime}$ by Proposition 5.1 part (2) and that $V \exp (-i \chi)=V^{\prime} \exp \left(-i \chi^{\prime}\right)$ by Proposition 5.4, part (2), it follows that

$$
w_{t_{0}}\left(t_{0}\right) \exp \left(-i \varphi_{t_{0}}\left(t_{0}\right)\right)=w_{t_{0}}^{\prime}\left(t_{0}\right) \exp \left(-i \varphi_{t_{0}}^{\prime}\left(t_{0}\right)\right)
$$

and therefore by Lemma $7.2,\left(w_{t_{0}}, \varphi_{t_{0}}\right)$ and $\left(w_{t_{0}}^{\prime}, \varphi_{t_{0}}^{\prime}\right)$ are gauge equivalent, namely

$$
w_{t_{0}}(t) \exp \left(-i \varphi_{t_{0}}(t)\right)=w_{t_{0}}^{\prime}(t) \exp \left(-i \varphi_{t_{0}}^{\prime}(t)\right)
$$

for all $t$ for which both solutions are defined.

We now take the limit $t_{0} \rightarrow \infty$ for fixed $t$ in (7.12). By Proposition 6.3 part (2), for fixed $t$, $\left(w_{t_{0}}, \varphi_{t_{0}}\right)$ and $\left(w_{t_{0}}^{\prime}, \varphi_{t_{0}}^{\prime}\right)$ converge respectively to $(w, \varphi)$ and $\left(w^{\prime}, \varphi^{\prime}\right)$ in $H^{k-1} \oplus Y^{\ell-1}$. By Lemma 3.1 , one can take the limit $t_{0} \rightarrow \infty$ in $(7.12)$, thereby obtaining $(7.6)$, so that $(w, \varphi)$ and $\left(w^{\prime}, \varphi^{\prime}\right)$ are gauge equivalent.

We can now define the wave operators for $u$. We recall from the heuristic discussion of Section 2 that we want to exploit the operator $\Omega_{0}$ defined in Definition 7.1 , reconstruct $u$ through the map $\Lambda$ defined by (7.5) and eliminate the arbitrariness in $\psi_{+}$by fixing $\psi_{+}=0$, thereby 
ensuring the injectivity of the wave operator for $u$.

Definition 7.4. We define the wave operator $\Omega$ as the map

$$
\Omega: u_{+} \rightarrow u=\left(\Lambda \circ \Omega_{0}\right)\left(F u_{+}, 0\right)
$$

from $F H^{k+(p+1) \vee 2}$ to $\mathcal{X}^{k}([T, \infty))$ for some $T, 1 \leq T<\infty$, where $k$ is the first element of an admissible pair, and $\Omega_{0}$ and $\Lambda$ are defined by Definition 7.1 and by (7.5).

The fact that $\Omega$ acts between the spaces indicated follows from Proposition 6.3 and Lemma 7.1.

\section{Proposition 7.4.}

(1) The map $\Omega$ is injective.

(2) If $0 \leq p \leq 2$, then $\mathcal{R}(\Omega)=\mathcal{R}\left(\Lambda \circ \Omega_{0}\right)$.

Proof. Part (1) follows from the fact that $\Omega_{0}$ is an injective map between gauge equivalence classes and that an equivalence class of asymptotic states contains at most one representative with $\psi_{+}=0$.

Part (2) follows from the fact that the gauge equivalence class of a given $\left(w_{+}, \psi_{+}\right)$actually contains an element with $\psi_{+}=0$, namely $\left(w_{+} \exp \left(-i \psi_{+}\right), 0\right)$, by Lemma 3.1.

Remark 7.2. Part (2) of Proposition 7.4 does not extend to the case $p \geq 3$ because in that case $\left(w_{+}, \psi_{+}\right) \in H^{k+p+1} \oplus Y^{\ell+1}$ does not imply that $w_{+} \exp \left(-i \psi_{+}\right) \in H^{k+p+1}$, so that the gauge equivalence class of a given $\left(w_{+}, \psi_{+}\right)$need not contain an element with $\psi_{+}=0$.

We now collect the information obtained for the solutions of the equation (1.1) so far constructed. The main result of this paper can be stated as follows.

Proposition 7.5. Let $n \geq 3,0<\mu \leq n-2$ and $0<\gamma \leq 1$. Let $(k, \ell)$ be an admissible pair. 
Let $p \geq 0$ be an integer with $(p+2) \gamma>1$. Let $u_{+} \in F H^{k+(p+1) \vee 2}$ and $a=\left|F u_{+}\right|_{k+(p+1) \vee 2}$. Let $W_{p}$ and $\phi_{p}$ be defined by (5.11) and Proposition 5.1 with $w_{+}=F u_{+}$. Then

(1) There exists $T, 1 \leq T<\infty$, and there exists a unique solution $u \in \mathcal{X}^{k}([T, \infty))$ of the equation (1.1) which can be represented as

$$
u=M D \exp (-i \varphi) w
$$

where $(w, \varphi)$ is a solution of the system (4.1) (4.2) such that $\left(w, h_{0}^{-1} \varphi\right) \in\left(\mathcal{C} \cap L^{\infty}\right)\left([T, \infty), H^{k} \oplus\right.$ $\left.Y^{\ell}\right)$ and such that

$$
\begin{gathered}
\left|w(t)-F u_{+}\right|_{k-1} h_{0}(t) \rightarrow 0 \\
\left|\varphi(t)-\phi_{p}(t)\right|_{\ell-1} \rightarrow 0
\end{gathered}
$$

when $t \rightarrow \infty$, where $h_{0}$ is defined by (3.19). The time $T$ can be defined by (6.14) with $b_{+}=0$.

(2) The solution is obtained as $u=\Omega\left(u_{+}\right)$where the map $\Omega$ is defined in Definition 7.4 . The map $\Omega$ is injective.

(3) The map $\Omega$ is continuous on the bounded sets of $F H^{k+(p+1) \vee 2}$ from the norm topology in $F H^{k+p-1}$ for $u_{+}$to the norm topology in $\mathcal{X}^{k-1}(I)$ and to the weak-* topology in $\mathcal{X}^{k}(I)$ for $u$ for any compact interval $I \subset[T, \infty)$, and to the weak topology in $M D H^{k}$ pointwise in $t$.

(4) The solution $u$ satisfies the following estimates for $t \geq T$

$$
\left\|<J(t)>^{k}\left(\exp \left(i \phi_{p}(t, x / t)\right) u(t)-M(t) D(t) F u_{+}\right)\right\|_{2} \leq A(a) P_{p}(t)
$$

for some estimating function $A(a)$, where $P_{p}(t)$ is defined by (3.31).

(5) Let $r$ satisfy $0 \leq \delta(r) \equiv n / 2-n / r \leq k \wedge n / 2, \delta(r)<n / 2$ if $k=n / 2$. Then u satisfies the following estimate

$$
\left\|u(t)-\exp \left(-i \phi_{p}(t, x / t)\right) M(t) D(t) F u_{+}\right\|_{r} \leq A(a) t^{-\delta(r)} P_{p}(t)
$$

Proof. Parts (1) (2) (3) follow from Proposition 6.3, Proposition 4.3, from Definition 7.4, Proposition 7.4 part (1), and Lemma 7.1.

Part (4). From the definition (7.2) of $J(t)$, from the commutation relation (7.3) and from Lemma 3.1, it follows that the LHS of (7.16) is estimated by

$$
\|\cdot\|_{2}=\left|\exp \left(i\left(\phi_{p}-\varphi\right)\right) w-F u_{+}\right|_{k} \leq\left|w-F u_{+}\right|_{k}+\left|\left(\exp \left(i\left(\phi_{p}-\varphi\right)\right)-1\right) w\right|_{k}
$$




$$
\leq\left|w-F u_{+}\right|_{k}+\left|\phi_{p}-\varphi\right|_{\ell-1}\left(1+\left|\phi_{p}-\varphi\right|_{\ell-1}\right)^{k-1}|w|_{k}
$$

The result now follows from the estimates (4.17) and (6.45) (6.46).

Part (5) follows from Part (4) and from the inequality

$$
\begin{aligned}
\|f\|_{r} & =t^{-\delta(r)}\left\|D^{*} M^{*} f\right\|_{r} \leq C t^{-\delta(r)}\left\|<\nabla>^{k} D^{*} M^{*} f\right\|_{2} \\
& =C t^{-\delta(r)}\left\|<J(t)>^{k} f\right\|_{2}
\end{aligned}
$$

which follows from the commutation relation (7.3) and from Sobolev inequalities.

Remark 7.3. In (7.16) and (7.17) one could replace $M D F u_{+}$by $U(t) u_{+}$since $U(t) u_{+}-$ $M D F u_{+}=O\left(t^{-1}\right)$ in the relevant norms. One could also replace $F u_{+}$by $W_{p}$, but this would not produce any improvement in the final estimates, since the main contribution of the difference between $u$ and its asymptotic form is that of the phase.

Finally, by combining Proposition 7.5 with the known results on the Cauchy problem for the equation (1.1) at finite times, one could extend the solutions $u$ to arbitrary finite times and define more standard wave operators $\Omega_{1}: u_{+} \rightarrow u(1)$ where $u=\Omega u_{+}$. We refer to I for the details.

Acknowledgements. One of us (G. V.) is grateful to Professor J. C. Saut for the hospitality at the Laboratoire d'Analyse Numérique et Equations aux Dérivés Partielles and to Professor D. Schiff for the hospitality at the Laboratoire de Physique Théorique.

\section{References}

[1] J. Derezinski, C. Gérard : Scattering theory of classical and quantum $N$-particle systems, Springer, Berlin, 1997.

[2] J. Ginibre, T. Ozawa : Long range scattering for nonlinear Schrödinger and Hartree equations in space dimension $n \geq 2$, Commun. Math. Phys. 151 (1993), 619-645. 
[3] J. Ginibre, G. Velo : On a class of nonlinear Schrödinger equations with nonlocal interaction, Math. Z. 170 (1980), 109-136.

[4] J. Ginibre, G. Velo : Long range scattering and modified wave operators for some Hartree type equations I, Rev. Math. Phys., to appear.

[5] N. Hayashi, E. I. Kaikina, P. I. Naumkin : On the scattering theory for the cubic nonlinear Schrödinger and Hartree type equations in one space dimension, Hokkaïdo Math. J., to appear.

[6] N. Hayashi, P. I. Naumkin : Asymptotics for large time of solutions to the nonlinear Schrödinger and Hartree equations, Am. J. Math. 120 (1998), 369-389.

[7] N. Hayashi, P. I. Naumkin : Scattering theory and large time asymptotics of solutions to Hartree type equations with a long range potential, preprint, 1997.

[8] N. Hayashi, P. I. Naumkin : Remarks on scattering theory and large time asymptotics of solutions to Hartree type equations with a long range potential, SUT J. of Math. 34 (1998), 13-24.

[9] N. Hayashi, P. I. Naumkin, T. Ozawa : Scattering theory for the Hartree equation, SIAM J. Math. Anal. 29 (1998), 1256-1267.

[10] N. Hayashi, Y. Tsutsumi : Scattering theory for Hartree type equations, Ann. IHP (Phys. Théor.) 46 (1987), 187-213.

[11] H. Nawa, T. Ozawa : Nonlinear scattering with nonlinear interaction, Commun. Math. Phys. 146 (1992), 259-275.

[12] T. Ozawa : Long range scattering for nonlinear Schrödinger equations in one space dimension, Commun. Math. Phys. 139 (1991), 479-493.

[13] D. R. Yafaev : Wave operators for the Schrödinger equation, Theor. Mat. Phys. 45 (1980), 992-998. 